\title{
Hitchin Fibrations on Two-Dimensional Moduli Spaces of Irregular Higgs Bundles with One Singular Fiber
}

Péter IVANICS ${ }^{\dagger}$, András I. STIPSICZ ${ }^{\dagger}$ and Szilárd SZABÓ $\ddagger$

$\dagger$ Rényi Institute of Mathematics, 1053 Budapest, Reáltanoda utca 13-15, Hungary

E-mail: ipe@math.bme.hu,stipsicz.andras@renyi.hu

$¥$ Budapest University of Technology and Economics, 1111 Budapest, Egry József utca 1, H épület, Hungary

E-mail:szabosz@math.bme.hu

Received February 02, 2019, in final form October 25, 2019; Published online November 04, 2019 https://doi.org/10.3842/SIGMA.2019.085

\begin{abstract}
We analyze and completely describe the four cases when the Hitchin fibration on a 2-dimensional moduli space of irregular Higgs bundles over $\mathbb{C} P^{1}$ has a single singular fiber. The case when the fiber at infinity is of type $I_{0}^{*}$ is further analyzed, and we give constructions of all the possible configurations of singular curves in elliptic fibrations having this type of singular fiber at infinity.
\end{abstract}

Key words: irregular Higgs bundles; Hitchin fibration; elliptic fibrations

2010 Mathematics Subject Classification: 14J27; 14H40; 14H60

\section{Introduction}

Let $D$ be an effective divisor in $\mathbb{C} P^{1}$ of total length 4 . Consider the (complex) 2-dimensional moduli spaces $\overline{\mathcal{M}^{\vec{\alpha}-s}\left(\mathbb{C} P^{1}, D, 2, d\right)}$ of rank 2 degree $d$ parabolically stable Higgs bundles over $\mathbb{C} P^{1}$ with irregular singularities of prescribed local form at $D$ and of 0 parabolic degree. When equipped with the Hitchin fibration, this space has been shown to be biregular to the complement of a singular fiber in an elliptic fibration on a rational elliptic surface (which complex surface is diffeomorphic to the 9-fold blow-up $\mathbb{C} P^{2} \#_{9} \overline{\mathbb{C} P}^{2}$ of the complex projective plane), see [14, Section 3.3] for the details of biregularity.

In the following we will describe all the cases when the Hitchin fibration on the moduli space has a single singular fiber. In these cases the fibration on the compactified space has exactly two singular fibers; fibrations with exactly two singular fibers have been extensively studied $[10,26]-$ indeed, the genus- 1 case (which is of central relevance in our subsequent studies) admits a simple classification scheme given in [26, Theorem 3.2]. In [26, Theorem 3.2] four cases and an infinite family of genus-1 fibrations with exactly two singular fibers have been encountered - since by [24, Section 4] all fibrations we will study arise from pencils (hence admit a section), the infinite family will not arise in our context.

We will distinguish five main cases, depending on the number of poles of the Higgs field (constrained by our assumption having a complex 2-dimensional moduli space):

1) there is one pole (which has to be of multiplicity 4),

2) there are two poles, each of multiplicity 2 ,

3) there are two poles, one with multiplicity 3 and the other with multiplicity 1 , 
4) there are three poles, one with multiplicity 2 and the further two with multiplicity 1 , and finally

5) there are four poles, each with multiplicity 1.

At each pole we need to distinguish further two cases, depending on whether the leading-order term of the polar part of the Higgs field at the pole is a regular semi-simple endomorphism (untwisted case), or has non-vanishing nilpotent part (twisted case). The third possible case, when the polar part is non-regular semi-simple, does not provide elliptic fibration, as shown by a simple calculation similar to [14, Lemma 5.6].

The polar parts will depend on some complex parameters, and our first aim is to determine those parameter values for which the Hitchin fibration on the moduli space has a unique singular fiber. These parameter values are complex numbers once we fix certain trivializations - for details see Section 2. The second aim of the paper is to provide explicit constructions for all the possibilities of configurations of singular fibers in case (5) of the above list (four logarithmic poles).

The spaces considered in this paper have a rich geometry. In addition to the Dolbeault algebraic structure that we focus on, they admit a de Rham algebraic structure parameterizing certain connections with irregular singularities (related to the Dolbeault space via non-abelian Hodge theory), and a Betti algebraic structure parameterizing representations of the fundamental group of the punctured surface together with Stokes data (related to de Rham space via the irregular Riemann-Hilbert correspondence). This picture was first studied by Hitchin [11] for regular objects, then by Simpson [21] in the tame case, subsequently extended to wild singularities by Biquard and Boalch [2]. In a series of papers, Boalch studied the moduli spaces of connections with irregular singularities on a curve and the corresponding wild character varieties. In particular, Boalch [5] (in the untwisted case) and Boalch and Yamakawa [6] (in the twisted case) defined the notion of an irregular curve, and showed that for a fixed irregular curve, parabolic weights and orbits of the residues in appropriate sense, one gets a Betti moduli functor (first spelled out in [3] in the untwisted case) whose corresponding coarse moduli space exists, and is given by a quasi-Hamiltonian quotient construction [1]. In the particular cases under study here, the above results imply that the moduli spaces we are looking at admit the structure of a smooth hyperKähler manifold of real dimension 4 . It is known $[4,7,16]$ that an open subset of each such space is in fact a quiver variety [17]. More precisely, it is a fiber of the semi-universal deformation of a Kleinian surface singularity, with an action of the corresponding Weyl group on the base of the deformation family. Another equivalent way of labelling these spaces that appears in the literature is by the type of the central fiber of this family.

The results of this paper, together with those of the previous papers $[13,14]$ of the same authors, will be applied [25] by the third author to prove the numerical version of the $P=W$ conjecture by de Cataldo, Hausel and Migliorini [8] and Simpson's geometric counterpart thereof [22] in the particular cases covered by our papers, namely those corresponding to the Painlevé systems. It turns out that these conjectures are easier to treat in the presence of a $\mathbb{C}^{\times}$-action. In the Painlevé cases (and more generally, if the moduli space is complex 2-dimensional), the existence of such an action implies that the Hitchin fibration admits a unique singular fiber. Therefore, one may hope that finding moduli spaces with a unique singular Hitchin fiber may be of use in the generalization of the $P=W$ conjecture.

Throughout, we will be interested in moduli spaces $\mathcal{M}^{\vec{\alpha}-s}\left(\mathbb{C} P^{1}, D, 2\right)$ of rank 2 parabolic Higgs bundles over $\mathbb{C} P^{1}$ with higher order poles at some divisor $D$, with prescribed local form near the points of the divisor. For details, see Section 2.1. The moduli space admits a natural decomposition

$$
\mathcal{M}^{\vec{\alpha}-s}\left(\mathbb{C} P^{1}, D, 2\right)=\coprod_{d \in \mathbb{Z}} \mathcal{M}^{\vec{\alpha}-s}\left(\mathbb{C} P^{1}, D, 2, d\right)
$$


according to the degree of the underlying vector bundle $\mathcal{E}$. In the cases where the data fixed to define $\mathcal{M}^{\vec{\alpha}-s}\left(\mathbb{C} P^{1}, D, 2, d\right)$ contains a regular non-semisimple (i.e., non-closed) adjoint orbit at some logarithmic points $t \in D$, we will equally study the partial compactification

$$
\overline{\mathcal{M}^{\vec{\alpha}-s}\left(\mathbb{C} P^{1}, D, 2, d\right)}
$$

parameterizing parabolic Higgs bundles with possibly non-regular residues at the points $t$, having the same characteristic polynomial. This functor differs from $\mathcal{M}^{\vec{\alpha}-s}\left(\mathbb{C} P^{1}, D, 2, d\right)$ in that instead of imposing the adjoint orbit of the residue of the Higgs field at such points $t$, we merely impose that the residue lie in the closure of the given non-closed orbit. Then, (1.1) is a stratified algebraic space: the top-dimensional stratum is $\mathcal{M}^{\vec{\alpha}-s}\left(\mathbb{C} P^{1}, D, 2, d\right)$, and the lower-dimensional strata are moduli spaces of parabolic logarithmic Higgs bundles with some regular and some non-regular residues having the same eigenvalues as fixed for the definition of $\mathcal{M}^{\vec{\alpha}-s}\left(\mathbb{C} P^{1}, D, 2, d\right)$.

\subsection{The case of one pole}

This case was treated in [13], where we gave a complete classification of singular fibers of the Hitchin fibration. In particular, in the untwisted case the fibration depends on the complex parameters

$$
a_{ \pm}, b_{ \pm}, c_{ \pm}, \lambda_{ \pm} \in \mathbb{C}, \quad a_{+} \neq a_{-},
$$

and the fiber at infinity is of type $\widetilde{E}_{7}$. The $a_{+}=a_{-}$case does not lead to an elliptic fibration, cf. [14, Lemma 5.6]. For the geometric meaning of these parameters, see Section 2.1.1; the various singular fibers are defined in Section 4. Let

$$
\Delta=\left(\left(b_{-}-b_{+}\right)^{2}-4\left(a_{-}-a_{+}\right)\left(c_{-}-c_{+}\right)\right)^{3}-432\left(a_{-}-a_{+}\right)^{4} \lambda_{+}^{2} .
$$

With these notations we have

Theorem 1.1 ([13, Theorem 1.1]). In this case the Hitchin fibration on the moduli space $\mathcal{M}^{\vec{\alpha}-s}\left(\mathbb{C} P^{1}, D, 2, d\right)$ admits a single singular fiber if and only if $\lambda_{+}=\lambda_{-}=0$ and $\Delta=0$. The single singular fiber is of type III.

In the twisted case we have complex parameters (described in detail Section 2.1.1)

$$
b_{-8}, \ldots, b_{-3} \in \mathbb{C}, \quad b_{-7} \neq 0,
$$

the fiber at infinity is $\widetilde{E}_{8}$. The case $b_{-7}=0$ does not give elliptic fibration [13, Section 2.3.2]. We introduce $\Gamma=\left(b_{-6}^{2}+4 b_{-5}\right)^{2}-24 b_{-7}\left(b_{-6} b_{-4}+2 b_{-3}\right)$ and we have

Theorem 1.2 ([13, Theorem 1.3]). In this case the Hitchin fibration on the moduli space $\mathcal{M}^{\vec{\alpha}-s}\left(\mathbb{C} P^{1}, D, 2, d\right)$ admits a single singular fiber if and only if $\Gamma=0$. The single singular fiber is of type II (i.e., a cusp fiber).

\subsection{The case of two poles}

Let us consider first the case when both multiplicities are equal to 2 . In this case a simple argument (see also [14, Theorems 2.1, 2.2, 2.3]) shows that the fiber at infinity is $I_{n}^{*}$ with $n \in\{2,3,4\}$ (depending on whether the leading order term is regular semi-simple or not at the two poles), hence in this case there is no Hitchin fibration on $\mathcal{M}^{\vec{\alpha}-s}\left(\mathbb{C} P^{1}, D, 2, d\right)$ with exactly one singular fiber (cf. Corollary 4.2).

In the case of multiplicities 3 and 1 on the two poles, we have more examples; indeed, if the pole with multiplicity 3 is untwisted and the other one is twisted, then the fibration 
depends on the complex parameters $a_{+}, a_{-}, b_{+}, b_{-}, \lambda_{+}, \lambda_{-}, b_{-1}$; their geometric significance is given in Section 2.1.2. We assume $a_{+} \neq a_{-}$because $a_{+}=a_{-}$does not lead elliptic fibration see [14, Remark 6.7]. In addition, the fibration also depends on so-called parabolic weights $\vec{\alpha}=\left\{\left(\alpha_{j}^{+}, \alpha_{j}^{-}\right)\right\}_{j=1,2}$. By taking

$$
A=a_{-}-a_{+}, \quad B=b_{-}-b_{+}, \quad L=\lambda_{-}-\lambda_{+},
$$

and defining $\Theta=4 A^{2}\left(B^{2}-6 A L\right)$, we get

Theorem 1.3 ([14, Theorem 2.5], [25, Lemma 2]). Suppose that we have two poles, one with multiplicity 3 and the other one with multiplicity 1. Assume furthermore that the multiplicity 3 pole is untwisted, while the other one is twisted. (In this case the fiber at infinity is of type $\widetilde{E}_{6}$.) Then the Hitchin fibration on $\overline{\mathcal{M}^{\vec{\alpha}-s}\left(\mathbb{C} P^{1}, D, 2, d\right)}$ has a unique singular fiber if and only if $\Theta=0$ and $L=0$. For generic parabolic weights, the single singular fiber is of type $I V$.

If the multiplicity 1 pole is untwisted, then according to [14, Theorems $2.4,2.6]$ we do not have examples for unique singular fibers in the Hitchin fibration on the moduli space. Finally, if both poles are twisted, then the natural parameters (described in Section 2.1.2) take the form $b_{-6}, b_{-5}, b_{-4}, b_{-3}, b_{-2}, b_{-1}$. Define $Q=8 b_{-5}\left(Q \neq 0\right.$ due to [14, Lemma 6.8] and $R=b_{-4}^{2}+4 b_{-3}$; with these notations in place, we have

Theorem 1.4 ([14, Theorem 2.7], [25, Lemma 2]). Suppose that the multiplicities of the poles are 3 and 1 , and both are twisted. In this case the fiber at infinity is of type $\widetilde{E}_{7}$, and the Hitchin fibration on $\overline{\mathcal{M}^{\vec{\alpha}-s}\left(\mathbb{C} P^{1}, D, 2, d\right)}$ admits a single singular fiber if and only if $R=0$. For generic parabolic weights, the single singular fiber is of type III.

\subsection{The case of three poles}

In this case one of the poles is of multiplicity 2 and the two others are both of multiplicity 1 . A simple argument shows that the fiber at infinity is either $I_{1}^{*}$ (when the polar part is untwisted at the pole of multiplicity 2) or $I_{2}^{*}$ (when the polar part is twisted at the pole of multiplicity 2). Since by [23, Section 6] elliptic fibrations on the rational elliptic surface with a singular fiber $I_{1}^{*}$ or $I_{2}^{*}$ have at least three singular fibers (cf. Corollary 4.2), we conclude

Theorem 1.5. In the case of three poles, the Hitchin fibration admits at least two singular fibers on the moduli space $\overline{\mathcal{M}^{\vec{\alpha}-s}}$.

\subsection{The case of four poles}

This is the most interesting case. It is not hard to see, that the fiber at infinity is an $I_{0}^{*}$ fiber, and (once again by [23, Section 6]) there is one case when the complement of this fiber admits a single singular fiber: when this other fiber is also of type $I_{0}^{*}$.

Before turning to the statement, we would like to point out a new feature in this case. In all previous cases the holomorphic structure on the fiber at infinity (which was either $\widetilde{E}_{8}, \widetilde{E}_{7}$ or $\widetilde{E}_{6}$ ) was unique. The holomorphic structure on $I_{0}^{*}$, however is not unique. All five $\mathbb{C} P^{1}$-components of $I_{0}^{*}$ can be equipped with a unique holomorphic structure, but the four points where the curves corresponding to the leaves of the graph describing $I_{0}^{*}$ (see Fig. 1) intersect the central rational curve determine a cross ratio, which is a holomorphic invariant. We can assume that the four points of intersections are at $0,1, \infty$ and $t \in \mathbb{C} P^{1}$; hence $t$ is the complex parameter (distinct from $0,1, \infty)$ determining the complex structure on $I_{0}^{*}$. The pencil on the Hirzebruch surface in this case is determined by four complex numbers $a, b, c, d$ (cf. Section 2.1.3 and equations (2.11) for their geometric role). The fibration again depends on a choice of parabolic weights $\vec{\alpha}=\left\{\left(\alpha_{j}^{+}, \alpha_{j}^{-}\right)\right\}_{j=1, \ldots, 4}$, see Section 3 . We assume that the sum of all these weights is an 
integer, and moreover that this integer is equal to the negative of the degree of the underlying vector bundle of the Higgs bundle. With these notations and assumptions, we have

Theorem 1.6. The pencil given by the above parameters $t, a, b, c, d$ provides an elliptic fibration with a single singular fiber (besides the $I_{0}^{*}$ fiber at infinity) if and only if

$$
c=a+(b-a-d) t+d t^{2} .
$$

For generic parabolic weights the single singular fiber of the partial compactification

$$
\overline{\mathcal{M}^{\vec{\alpha}-s}\left(\mathbb{C} P^{1}, D, 2, d\right)}
$$

introduced in (1.1) is of type $I_{0}^{*}$.

The singular fibers of Hitchin fibrations in cases of one or two poles have been completely determined in $[13,14]$ in terms of the parameters. In principle a similar analysis can be carried out in the remaining cases - in Section 6 we will restrict ourselves to provide examples of all possible singular fiber configurations when we have four poles. For the list of these possibilities, see Table 1 in Section 6. Here we do not determine the various configurations in terms of the parameters. By providing various constructions and examples, we prove:

Theorem 1.7. Any configuration of singular fibers on the complement of an $I_{0}^{*}$ fiber in a rational elliptic surface arises as the set of singular fibers of a Hitchin fibration.

The paper is organized as follows. In Section 2 we collect some facts and definitions of Higgs bundles and the Hitchin map, relevant to our present investigation. We also include the necessary stability analysis here. Section 4 is devoted to list background material regarding elliptic fibrations, and in Section 5 we describe the proofs of the statements about Hitchin fibrations with unique singular fibers given in this introduction. Finally in Section 6 we describe a number of constructions for elliptic fibrations with one of the singular fibers being $I_{0}^{*}$ (relevant in the case of four poles in the above discussion), hence proving Theorem 1.7. In these constructions we pay special attention to the further singular fibers of the resulting fibrations.

\section{Preliminaries}

In this paper we will deal with certain meromorphic Higgs bundles over $\mathbb{C} P^{1}$ with structure group $\mathrm{GL}(2, \mathbb{C})$. In the sequel we let $D$ be an effective divisor over $\mathbb{C} P^{1}$ and denote by $K_{\mathbb{C} P^{1}}=\boldsymbol{\Omega}_{\mathbb{C} P^{1}}^{1}$ the canonical bundle of $\mathbb{C} P^{1}$.

Definition 2.1. A meromorphic Higgs bundle with polar divisor $D$ (or a $K(D)$-pair of rank 2 ) over $\mathbb{C} P^{1}$ consists of a pair $(\mathcal{E}, \theta)$ where $\mathcal{E}$ is a holomorphic rank 2 vector bundle over $\mathbb{C} P^{1}$ and

$$
\theta: \mathcal{E} \rightarrow \mathcal{E} \otimes K_{\mathbb{C} P^{1}}(D)
$$

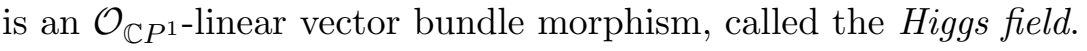

From now on, we assume that the total length of $D$ is equal to 4 :

$$
D=m_{1} t_{1}+\cdots+m_{k} t_{k}, \quad m_{1}+\cdots+m_{k}=4,
$$

where $1 \leq k \leq 4,1 \leq m_{j}$ and $t_{j} \in \mathbb{C} P^{1}$ are distinct points for $1 \leq j \leq k$. We have an $\mathcal{O}_{\mathbb{C} P^{1} \text {-linear isomorphism of line bundles }}$

$$
K_{\mathbb{C} P^{1}}(D) \cong \mathcal{O}_{\mathbb{C} P^{1}}(2) .
$$

We denote 
- by $\mathbb{F}_{2}$ the Hirzebruch surface obtained by the projectivization of $K_{\mathbb{C} P^{1}}(D)$, and by $p: \mathbb{F}_{2} \rightarrow$ $\mathbb{C} P^{1}$ the ruling coming from this construction,

- by $\mathcal{O}_{\mathbb{F}_{2} \mid \mathbb{C} P^{1}}(1)$ the relative ample bundle of $p$ and by $\mathcal{O}_{\mathbb{F}_{2} \mid \mathbb{C} P^{1}}(n)=\mathcal{O}_{\mathbb{F}_{2} \mid \mathbb{C} P^{1}}(1)^{\otimes n}$ its tensor powers,

- by $\zeta \in H^{0}\left(\mathbb{F}_{2}, p^{*} K_{\mathbb{C} P^{1}}(D) \otimes \mathcal{O}_{\mathbb{F}_{2} \mid \mathbb{C} P^{1}}(1)\right)$ and $\xi \in H^{0}\left(\mathbb{F}_{2}, \mathcal{O}_{\mathbb{F}_{2} \mid \mathbb{C} P^{1}}(1)\right)$ the canonical sections,

- and by $I_{\mathcal{E}}$ the identity endomorphism of $\mathcal{E}$.

Let $(\mathcal{E}, \theta)$ be a $K(D)$-pair of rank 2 over $\mathbb{C} P^{1}$.

Definition 2.2. The characteristic polynomial of $\theta$ is the section

$$
\chi_{\theta}(\zeta)=\operatorname{det}\left(\zeta I_{\mathcal{E}}-\xi p^{*} \theta\right)=\zeta^{2}+\zeta \xi F_{\theta}+\xi^{2} G_{\theta} \in H^{0}\left(\mathbb{F}_{2}, p^{*} K_{\mathbb{C} P^{1}}(D)^{\otimes 2} \otimes \mathcal{O}_{\mathbb{F}_{2} \mid \mathbb{C} P^{1}}(2)\right) .
$$

Here

$$
F_{\theta} \in H^{0}\left(\mathbb{C} P^{1}, K_{\mathbb{C} P^{1}}(D)\right), \quad G_{\theta} \in H^{0}\left(\mathbb{C} P^{1}, K_{\mathbb{C} P^{1}}(D)^{\otimes 2}\right)
$$

are called the characteristic coefficients of $\theta$. Elements of $H^{0}\left(\mathbb{C} P^{1}, K_{\mathbb{C} P^{1}}(D)\right)$ will be called meromorphic differentials and elements of $H^{0}\left(\mathbb{C} P^{1}, K_{\mathbb{C} P^{1}}(D)^{\otimes 2}\right)$ meromorphic quadratic differentials. The spectral curve of $(\mathcal{E}, \theta)$ is the curve in $\mathbb{F}_{2}$ defined by the equation $\chi_{\theta}(\zeta)=0$. The Hitchin base is the vector space

$$
\mathcal{B}=H^{0}\left(\mathbb{C} P^{1}, K_{\mathbb{C} P^{1}}(D)\right) \oplus H^{0}\left(\mathbb{C} P^{1}, K_{\mathbb{C} P^{1}}(D)^{\otimes 2}\right)
$$

containing all possible characteristic coefficients of rank $2 K(D)$-pairs.

It follows from the definition and (2.2) that the characteristic coefficients $F_{\theta}, G_{\theta}$ may be considered as global sections of $\mathcal{O}_{\mathbb{C} P^{1}}(2)$ and of $\mathcal{O}_{\mathbb{C} P^{1}}(4)$ respectively, so we have

$$
\operatorname{dim}_{\mathbb{C}} \mathcal{B}=8 .
$$

Remark 2.3. In Section 6 we will take a converse approach by setting $F_{\theta}=0$ and considering sections

$$
\sigma \in H^{0}\left(\mathbb{C} P^{1}, \mathcal{O}_{\mathbb{C} P^{1}}(4)\right)
$$

(corresponding to the coefficient $G_{\theta}$ ); we then choose the points $t_{j}$ (or equivalently, the fibers $\left.F_{j}=p^{-1}\left(t_{j}\right)\right)$ appropriately in order to obtain various singular fibers.

Let us denote by

$$
\mathcal{M}\left(\mathbb{C} P^{1}, D, 2\right)
$$

the moduli stack of $K(D)$-pairs of rank 2 over $\mathbb{C} P^{1}$. It decomposes as

$$
\mathcal{M}\left(\mathbb{C} P^{1}, D, 2\right)=\coprod_{d \in \mathbb{Z}} \mathcal{M}\left(\mathbb{C} P^{1}, D, 2, d\right)
$$

where $\mathcal{M}\left(\mathbb{C} P^{1}, D, 2, d\right)$ is the moduli stack of $K(D)$-pairs of rank 2 over $\mathbb{C} P^{1}$ satisfying $\operatorname{deg}(\mathcal{E})$ $=d$.

Definition 2.4. The Hitchin map is the morphism

$$
\begin{aligned}
h: \mathcal{M}\left(\mathbb{C} P^{1}, D, 2\right) & \rightarrow \mathcal{B}, \\
(\mathcal{E}, \theta) & \mapsto\left(F_{\theta}, G_{\theta}\right)
\end{aligned}
$$

associating to a Higgs bundle its characteristic coefficients. 
For generic $(F, G) \in \mathcal{B}$, the curve $\Sigma_{(F, G)}$ defined by the equation

$$
\zeta^{2}+\zeta \xi F+\xi^{2} G
$$

is smooth, and for every $d \in \mathbb{Z}$ the fiber of

$$
h: \mathcal{M}\left(\mathbb{C} P^{1}, D, 2, d\right) \rightarrow \mathcal{B}
$$

is a torsor over the $\operatorname{Jacobian} \operatorname{Jac}\left(\Sigma_{(F, G)}\right)$ of $\Sigma_{(F, G)}$. When the curve $\Sigma_{(F, G)}$ is not regular, then $\operatorname{Jac}\left(\Sigma_{(F, G)}\right)$ is non-compact, and for every $d$ the fiber $h^{-1}(F, G)$ is (non-canonically) isomorphic to a certain compactification of $\operatorname{Jac}\left(\Sigma_{(F, G)}\right)$. In case $\Sigma_{(F, G)}$ is integral, the suitable compactification of $\operatorname{Jac}\left(\Sigma_{(F, G)}\right)$ is the moduli space of torsion-free sheaves of rank 1 and given degree over $\Sigma_{(F, G)}$. On the other hand, in case $\Sigma_{(F, G)}$ is reducible or non-reduced, the corresponding fiber of (2.4) is not of finite type, and in order to get a moduli scheme of finite type one needs to impose a stability condition on the objects. Stability conditions naturally arise from parabolic structures on meromorphic Higgs bundles; we will return to describing moduli spaces of stable objects and the fibers of the Hitchin map on these moduli spaces once we have the assumptions and notations concerning the local behaviour of a meromorphic Higgs field near the points of $D$.

\subsection{Parameters and their geometric significance}

Our investigation is based on the local description of spectral curves on the Hirzebruch surface $\mathbb{F}_{2}$ of degree 2. Recall from equation (2.1) that $D=m_{1} t_{1}+\cdots+m_{k} t_{k}$, with $m_{1}+\cdots+m_{k}=4$.

If we are in the case of one pole with multiplicity 4 at $t_{1}$, then we introduce two local charts on $\mathbb{C} P^{1}: U$ with $u \in \mathbb{C}$ (where $\{u=0\}=[0: 1]=t_{1}$ ) and $V$ with $v \in \mathbb{C}$ (where $\{v=0\}=[1: 0]$ ). The bundle $K_{\mathbb{C} P^{1}}\left(4 t_{1}\right)$ admits the trivializing sections $\kappa_{U}$ over $U$ and $\kappa_{V}$ over $V$ :

$$
\kappa_{U}=\frac{\mathrm{d} u}{u^{4}}, \quad \kappa_{V}=\mathrm{d} v .
$$

If we are in the case of at least two poles, then we introduce two local charts on $\mathbb{C} P^{1}: U$ with $u \in \mathbb{C}\left(\right.$ where $\left.\{u=0\}=[0: 1]=t_{1}\right)$ and $V$ with $v \in \mathbb{C}\left(\right.$ where $\left.\{v=0\}=[1: 0]=t_{k}\right)$. We also denote by $t_{i}$ the representation of a pole in the local chart $U$, we refer $t_{1}$ as $0, t_{k}$ as $\infty$ and $t_{i}^{-1}$ as the image of $t_{i} \in \mathbb{C} P^{1}$ in chart $V$. The bundle $K_{\mathbb{C} P^{1}}(D)$ admits the following trivializing sections:

$$
\kappa_{U}=\frac{\mathrm{d} u}{\prod_{i=2}^{k-1}\left(u-t_{i}\right)^{m_{i}}}, \quad \kappa_{V}=\frac{\mathrm{d} v}{\prod_{i=2}^{k-1}\left(v-t_{i}^{-1}\right)^{m_{i}}} .
$$

The conversion from $\kappa_{U}$ to $\kappa_{V}$ is the following (if the total multiplicity is 4 ):

$$
\kappa_{U}=-\frac{v^{2}}{\prod_{i=2}^{k-1}\left(-t_{i}\right)^{m_{i}}} \kappa_{V} .
$$

The trivialization $\kappa_{j}$ induces a trivialization $\kappa_{j}^{2}$ on $K_{\mathbb{C} P^{1}}(D)^{\otimes 2}, j=U, V$. Moreover, (recalling that $\zeta$ denotes the canonical section of $\left.p^{*} K_{\mathbb{C} P^{1}}(D) \otimes \mathcal{O}_{\mathbb{F}_{2} \mid \mathbb{C} P^{1}}(1)\right)$ we can introduce coordinates $w$ in $p^{-1}(U)$ and $z$ in $p^{-1}(V)$ by

$$
\zeta=w \otimes \kappa_{U}=z \otimes \kappa_{V} .
$$

Choose a suitable trivialization of $\mathcal{E}$. Consider an irregular Higgs bundle $(\mathcal{E}, \theta)$ in the $\kappa_{j}$ trivializations $(j=U, V)$ and the chosen trivialization of $\mathcal{E}$. The local forms of $\theta$ near $t_{i}$ 's are the following: 
- the case of $D=4 t_{1}$ :

$$
\theta=\sum_{n \geq-4} A_{n} u^{n} \otimes \mathrm{d} u
$$

- the case of $D=2 t_{1}+2 t_{2}$ is not of interest, because we concentrate on the cases with two singular fibers, but according to [14, Theorems 2.1, 2.2, 2.3], $D=2 t_{1}+2 t_{2}$ to leads at least three singular fibers,

- the case of $D=3 t_{1}+1 t_{2}$ :

$$
\theta=\sum_{n \geq-3} A_{n} u^{n} \otimes \mathrm{d} u \quad \text { and } \quad \theta=\sum_{n \geq-1} B_{n} v^{n} \otimes \mathrm{d} v
$$

- the case of $D=2 t_{1}+1 t_{2}+1 t_{3}$ is not relevant: a simple geometric argument similar to Example 4.3 shows that in this case the elliptic fibration contains a fiber of type $I_{1}^{*}$ or $I_{2}^{*}$, and according to Corollary 4.2 this case can not lead to exactly two singular fibers,

- the case of $D=1 t_{1}+1 t_{2}+1 t_{3}+1 t_{4}$. Near one of the $t_{i}(i=1,2,3)$ the local form is

$$
\theta=\sum_{n \geq-1} A_{n, i}\left(u-t_{i}\right)^{n} \otimes \mathrm{d} u
$$

and near $t_{4}=\infty$ :

$$
\theta=\sum_{n \geq-1} A_{n, 4} v^{n} \otimes \mathrm{d} v
$$

where $A_{n}, B_{n}, A_{n, i} \in \mathfrak{g l}(2, \mathbb{C})$.

The coefficient $A_{-1, i}$ in above expression is called the residue of $\theta$ at $t_{i}$, with respect to the chosen trivialization of $\mathcal{E}$. The residue of $\theta$ at $t_{i}$ is denoted by $\operatorname{res}_{t_{i}}(\theta)$. It is a well-defined endomorphism of $\left.\mathcal{E}\right|_{t_{i}}(i=1, \ldots, 4)$.

The spectral curve $\left(\chi_{\theta}(\zeta)=0\right)$ has an expansion near $t_{i}$ in which these parameters have a geometric meaning. The lowest index matrices $A_{n}, B_{n}$ and $A_{n, i}$ in equations (2.6), (2.7) and (2.8) encode the base locus of a pencil.

Let us consider the local forms separated according to the number of poles and their multiplicities.

\subsection{1 $D=4 t_{1}$}

The matrices $A_{-3}, A_{-2}$ and $A_{-1}$ in equation (2.6) encode the tangent, the second and third derivative of the curve in the pencil. If the leading order term is a regular semi-simple endomorphism (untwisted case) then the local form in the trivialization $\kappa_{U}$ of $K_{\mathbb{C} P^{1}}$ and the chosen trivialization of $\mathcal{E}$ is

$$
\theta=\left[\left(\begin{array}{cc}
a_{+} & 0 \\
0 & a_{-}
\end{array}\right) u^{-4}+\left(\begin{array}{cc}
b_{+} & 0 \\
0 & b_{-}
\end{array}\right) u^{-3}+\left(\begin{array}{cc}
c_{+} & 0 \\
0 & c_{-}
\end{array}\right) u^{-2}+\left(\begin{array}{cc}
\lambda_{+} & 0 \\
0 & \lambda_{-}
\end{array}\right) u^{-1}+O(1)\right] \otimes \mathrm{d} u .
$$

These matrices provide the parameters (U) in Theorem 1.1.

If the leading order term has non-vanishing nilpotent part (twisted case) then the local form in the trivialization $\kappa_{U}$ is

$$
\theta=\left[\left(\begin{array}{cc}
b_{-8} & 1 \\
0 & b_{-8}
\end{array}\right) u^{-4}+\left(\begin{array}{cc}
0 & 0 \\
b_{-7} & b_{-6}
\end{array}\right) u^{-3}+\left(\begin{array}{cc}
0 & 0 \\
b_{-5} & b_{-4}
\end{array}\right) u^{-2}+\left(\begin{array}{cc}
0 & 0 \\
b_{-3} & b_{-2}
\end{array}\right) u^{-1}+O(1)\right] \otimes \mathrm{d} u,
$$

with $b_{-7} \neq 0$. These matrices provide the parameters $(\mathrm{T})$ in Theorem 1.2. 


\subsection{2 $D=3 t_{1}+1 t_{2}$}

The matrices $A_{-2}$ and $A_{-1}$ in equation (2.7) encode the slope of the tangent line of a pencil and the second derivative of the curve in the pencil.

If the pole with multiplicity 3 (i.e., $t_{1}$ ) is untwisted then the local form in the trivialization $\kappa_{U}$ (near $t_{1}$ ) and the chosen trivialization of $\mathcal{E}$ :

$$
\theta=\left[\left(\begin{array}{cc}
a_{+} & 0 \\
0 & a_{-}
\end{array}\right) u^{-3}+\left(\begin{array}{cc}
b_{+} & 0 \\
0 & b_{-}
\end{array}\right) u^{-2}+\left(\begin{array}{cc}
\lambda_{+} & 0 \\
0 & \lambda_{-}
\end{array}\right) u^{-1}+O(1)\right] \otimes \mathrm{d} u .
$$

If the multiplicity 3 pole is twisted, then

$$
\theta=\left[\left(\begin{array}{cc}
b_{-6} & 1 \\
0 & b_{-6}
\end{array}\right) u^{-3}+\left(\begin{array}{cc}
0 & 0 \\
b_{-5} & b_{-4}
\end{array}\right) u^{-2}+\left(\begin{array}{cc}
0 & 0 \\
b_{-3} & b_{-2}
\end{array}\right) u^{-1}+O(1)\right] \otimes \mathrm{d} u,
$$

with $b_{-5} \neq 0$.

The matrix $B_{-1}$ contains the base locus of the pencil in the trivialization $\kappa_{V}$ and the chosen trivialization of $\mathcal{E}$. If the pole $t_{2}$ is untwisted, then

$$
\theta=\left[\left(\begin{array}{cc}
\mu_{+} & 0 \\
0 & \mu_{-}
\end{array}\right) v^{-1}+O(1)\right] \otimes \mathrm{d} v
$$

If the pole $t_{2}$ is twisted, then up to holomorphic gauge transformation

$$
\theta=\left[\left(\begin{array}{cc}
b_{-1} & \varepsilon \\
0 & b_{-1}
\end{array}\right) v^{-1}+O(1)\right] \otimes \mathrm{d} v
$$

for $\varepsilon \in \mathbb{C}$. These parameters appear in Theorems 1.3 and 1.4 .

\subsection{3 $D=1 t_{1}+1 t_{2}+1 t_{3}+1 t_{4}$}

The matrices in equations (2.8) only encode the base locus of the pencil in the four distinguished fibers at $t_{1}=0, t_{2}=1, t_{3}=t$ and $t_{4}=\infty \in \mathbb{C} P^{1}$.

If one of the poles $t_{i}(i=1, \ldots, 4)$ is untwisted then the matrix from the local form in the trivialization $\kappa_{j}(j=U, V)$ and the chosen trivialization of $\mathcal{E}$ is the following:

$$
A_{-1, i}=\left(\begin{array}{cc}
a_{+, i} & 0 \\
0 & a_{-, i}
\end{array}\right)
$$

where we assume that $a_{+, i} \neq a_{-, i}$, otherwise a similar computation as in [14, Lemma 5.6] shows that the fibration is not elliptic.

Similarly, if one of the poles $t_{i}(i=1, \ldots, 4)$ is twisted then the matrix has non-trivial nilpotent part. In the trivialization $\kappa_{j}(j=U, V)$ and the chosen trivialization of $\mathcal{E}$ :

$$
A_{-1, i}=\left(\begin{array}{cc}
a_{i} & \varepsilon \\
0 & a_{i}
\end{array}\right)
$$

for $\varepsilon \in \mathbb{C}$.

For simplicity we introduce new notations for the parameters appearing above:

$$
\begin{aligned}
& a_{ \pm}:=a_{ \pm, 1}, \\
& b_{ \pm}:=a_{ \pm, 2}, \\
& c_{ \pm}:=a_{ \pm, 3}, \\
& d_{ \pm}:=a_{ \pm, 4},
\end{aligned}
$$




$$
\begin{aligned}
a & :=a_{1}, \\
b & :=a_{2}, \\
c & :=a_{3}, \\
d & :=a_{4} .
\end{aligned}
$$

The parameters $a, b, c$ and $d$ which appear in the Theorem 1.6 encode the base locus in the distinguished fibers.

\section{Higgs bundles with non-reduced spectral curve}

Let $(\mathcal{E}, \theta)$ be a meromorphic Higgs bundle of rank 2 over $\mathbb{C} P^{1}$ with divisor $D$. Parabolic stability for Higgs fields with higher-order poles has been investigated in $[13,14]$. In this subsection we assume that the multiplicity $m_{j}$ is equal to 1 for all $j$, i.e., that

$$
D=t_{1}+\cdots+t_{4}
$$

for some distinct points $t_{j}(j=1,2,3,4)$.

Definition 3.1. A compatible quasi-parabolic structure is the choice of a 1-dimensional eigenspace $\left.\ell_{j} \subset \mathcal{E}\right|_{t_{j}}$ of $\operatorname{res}_{t_{j}}(\theta)$ for all $1 \leq j \leq 4$. A compatible parabolic structure consists of a compatible quasi-parabolic structure and a choice of a pair of rational numbers $\left(\alpha_{j}^{+}, \alpha_{j}^{-}\right)$ (called parabolic weights) satisfying

$$
0 \leq \alpha_{j}^{-}<\alpha_{j}^{+}<1
$$

for each $j$. The parabolic degree of $\mathcal{E}$ is defined by the formula

$$
\operatorname{deg}_{\vec{\alpha}}(\mathcal{E})=\operatorname{deg}(\mathcal{E})+\sum_{j=1}^{4}\left(\alpha_{j}^{+}+\alpha_{j}^{-}\right) .
$$

A rank 1 meromorphic Higgs subbundle of $(\mathcal{E}, \theta)$ is a pair $\left(\mathcal{F},\left.\theta\right|_{\mathcal{F}}\right)$ where $\mathcal{F}$ is a rank 1 subbundle of $\mathcal{E}$ such that the restriction $\left.\theta\right|_{\mathcal{F}}$ maps $\mathcal{F}$ into $\mathcal{F} \otimes K_{\mathbb{C} P^{1}}(D)$.

Let $\left(\mathcal{E}, \theta,\left\{\ell_{j}\right\}_{j=1}^{4}\right)$ be a meromorphic Higgs bundle of rank 2 over $\mathbb{C} P^{1}$ with a compatible quasi-parabolic structure, and with given parabolic weights $\left\{\left(\alpha_{j}^{+}, \alpha_{j}^{-}\right)\right\}_{j=1}^{4}$. For any rank 1 meromorphic Higgs subbundle $\left(\mathcal{F},\left.\theta\right|_{\mathcal{F}}\right)$ of $(\mathcal{E}, \theta)$, the fiber $\left.\mathcal{F}\right|_{t_{j}}$ is preserved by $\operatorname{res}_{t_{j}}(\theta)$. If $\left.\mathcal{F}\right|_{t_{j}}=\ell_{j}$, then we set

$$
\alpha_{j}(\mathcal{F})=\alpha_{j}^{+},
$$

otherwise we set

$$
\alpha_{j}(\mathcal{F})=\alpha_{j}^{-} .
$$

Finally, we define

$$
\operatorname{deg}_{\vec{\alpha}}(\mathcal{F})=\operatorname{deg}(\mathcal{F})+\sum_{j=1}^{4} \alpha_{j}(\mathcal{F}) .
$$

Definition 3.2. $\left(\mathcal{E}, \theta,\left\{\ell_{j}\right\}_{j=1}^{4}\right)$ is $\vec{\alpha}$-stable if for any rank 1 meromorphic Higgs subbundle $\left(\mathcal{F},\left.\theta\right|_{\mathcal{F}}\right)$ we have

$$
\operatorname{deg}_{\vec{\alpha}}(\mathcal{F})<\frac{\operatorname{deg}_{\vec{\alpha}}(\mathcal{E})}{2} .
$$


Moreover, $\left(\mathcal{E}, \theta,\left\{\ell_{j}\right\}_{j=1}^{4}\right)$ is $\vec{\alpha}$-semistable if for any rank 1 meromorphic Higgs subbundle $\left(\mathcal{F},\left.\theta\right|_{\mathcal{F}}\right)$ we have

$$
\operatorname{deg}_{\vec{\alpha}}(\mathcal{F}) \leq \frac{\operatorname{deg}_{\vec{\alpha}}(\mathcal{E})}{2} .
$$

Let $M^{\vec{\alpha}-s}$ and $M^{\vec{\alpha}-s s}$ be the functors

$$
\text { Schemes } \rightarrow \text { Sets }
$$

associating to a scheme $S$ the set of $S$-equivalence classes of holomorphic vector-bundles

$$
\mathcal{E} \rightarrow \mathbb{C} P^{1} \times S
$$

endowed with a morphism

$$
\theta: \mathcal{E} \rightarrow \mathcal{E} \otimes p_{1}^{*} K_{\mathbb{C} P^{1}}
$$

(where $p_{1}: \mathbb{C} P^{1} \times S \rightarrow \mathbb{C} P^{1}$ stands for the first projection) and with filtrations

$$
\left.\ell_{j} \subset \mathcal{E}\right|_{\left\{t_{j}\right\} \times S},
$$

where $\ell_{j}$ is a line-subbundle preserved by $\operatorname{res}_{\left\{t_{j}\right\} \times S}(\theta)$, and such that for any geometric point $s \in S$ the triple $\left(\mathcal{E}, \theta,\left\{\ell_{j}\right\}_{j=1}^{4}\right)$ defines an $\vec{\alpha}$-stable (respectively, $\vec{\alpha}$-semistable) meromorphic Higgs bundle of rank 2 over $\mathbb{C} P^{1}$ with a compatible parabolic structure and local form of the Higgs field specified in Section 2.1; for simplicity of the notation, we do not include the parameters $a, b, \ldots$ in the notation of the functors, but we tacitly fix them.

Theorem 3.3 ([19]). There exist quasi-projective coarse moduli schemes

$$
\mathcal{M}^{\vec{\alpha}-s}\left(\mathbb{C} P^{1}, D, 2\right) \subseteq \mathcal{M}^{\vec{\alpha}-s s}\left(\mathbb{C} P^{1}, D, 2\right)
$$

for the functors $M^{\vec{\alpha}-s}$ and $M^{\vec{\alpha}-s s}$, respectively.

The Hitchin map introduced in Definition 2.4 induces morphisms

$$
h: \overline{\mathcal{M}^{\vec{\alpha}-s}\left(\mathbb{C} P^{1}, D, 2, d\right)} \rightarrow \mathcal{B},
$$

and for any $(F, G) \in \mathcal{B}$ we denote by

$$
\overline{\mathcal{M}^{\vec{\alpha}-s}\left(\mathbb{C} P^{1}, D, 2, d\right)}(F, G)
$$

its fiber over $(F, G)$.

In $[13,14]$ we studied stability of Higgs bundles with spectral curves such that the curve in the associated elliptic fibration is of type $I_{n}(1 \leq n \leq 3), I I, I I I$ or $I V$. The only new case that appears in the cases treated here is that of a spectral curve $\Sigma$ such that the curve in the associated elliptic fibration is of type $I_{0}^{*}=\tilde{D}_{4}$. As we will see in Lemma 4.4, this case only appears if the spectral curve $\Sigma$ is a section of $p: \mathbb{F}_{2} \rightarrow \mathbb{C} P^{1}$ taken with multiplicity two. From now on, we assume that $\Sigma$ is of this type, and in addition that

$$
\operatorname{deg}_{\vec{\alpha}}(\mathcal{E})=0
$$

Up to tensoring with a rank 1 meromorphic Higgs bundle, the curve $\Sigma=\Sigma_{(0,0)}$ is the image of the 0 -section $F=0, G=0$. 
Assumption 3.4. From now on we only consider Higgs bundles satisfying

$$
\operatorname{deg}_{\vec{\alpha}}(\mathcal{E})=0 .
$$

This assumption implies that

$$
\sum_{j=1}^{4}\left(\alpha_{j}^{+}+\alpha_{j}^{-}\right) \in \mathbb{Z},
$$

so the space of possible parabolic weights belong to a finite union of affine hyperplanes in $[0,1)^{8}$. We will say that the parabolic weights are generic if for any choice of $\varepsilon_{j} \in\{+,-\}$ we have

$$
\sum_{j=1}^{4} \alpha_{j}^{\varepsilon_{j}} \notin \mathbb{Z} .
$$

Generic parabolic weights belong to the complement of finitely many proper affine subspaces of the affine hyperplanes of all possible parabolic weights. For generic parabolic weights, $\vec{\alpha}$-semistability is equivalent to $\vec{\alpha}$-stability, because the parabolic degree of an invariant subobject $\mathcal{F}$ is never an integer.

Proposition 3.5. For generic parabolic weights the Hitchin fiber

$$
{\overline{\mathcal{M}_{t}^{\vec{\alpha}-s}\left(\mathbb{C} P^{1}, D, 2, d\right)}}_{(0,0)}
$$

over $(0,0) \in \mathcal{B}$ is of type $I_{0}^{*}$.

Proof. It follows from [2, Theorem 0.2] that the moduli space in the assertion is a smooth complete hyperKähler manifold. Moreover, according to [25, Lemma 1], the Hitchin map defined on the moduli space is proper. It is well-known that the generic Hitchin fibers are elliptic curves; indeed, they are (up to a degree twist) the Jacobians of the elliptic curves appearing in the pencil of spectral curves to be studied in Example 4.3. Now, the elliptic fibration of Example 4.3 admits a central fiber of type $I_{0}^{*}$, therefore the monodromy matrix of the pencil of spectral curves is conjugate to

$$
\left(\begin{array}{cc}
-1 & 0 \\
0 & -1
\end{array}\right)
$$

It follows from this that the monodromy matrix of its dual fibration is also conjugate to the same matrix. As the monodromy matrix uniquely determines the Kodaira type of a singular fiber in an elliptic surface, we deduce that the only singular fiber of the Hitchin map is of type $I_{0}^{*}$ too.

Alternatively, it follows from [7] that an open subset of the moduli space is a smooth quiver variety associated to the quiver $D_{4}^{(1)}$ and dimension vector equal to the positive minimal integer generator of the radical of the associated quadratic form. This quiver variety is isomorphic to a minimal resolution of the surface singularity of type $D_{4}$. The exceptional divisors of this resolution lie in the singular Hitchin fiber, so the singular Hitchin fiber contains a curve of type $D_{4}$; the only Kodaira fiber containing a curve of this type is $I_{0}^{*}$.

\section{Singular fibers in elliptic fibrations}

An elliptic fibration is a map $\pi: X \rightarrow C$ where $X$ is a compact complex surface, $C$ is a compact complex curve and the generic fiber is an elliptic curve, i.e., smoothly diffeomorphic to the 2torus. In the following we will consider only those elliptic surfaces which admit a section, i.e., 


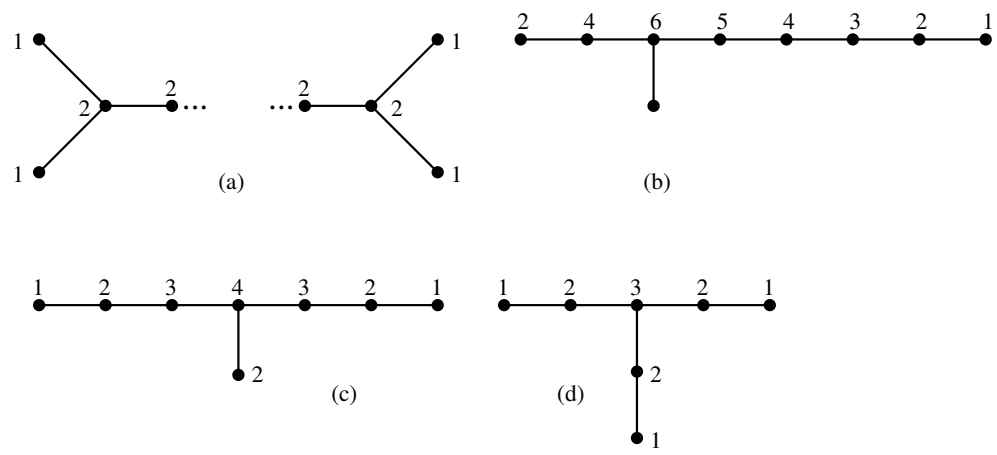

Figure 1. Plumbings of singular fibers of types (a) $I_{n}^{*}$, (b) $\tilde{E}_{8}$, (c) $\tilde{E}_{7}$, and (d) $\tilde{E}_{6}$. Integers next to vertices indicate the multiplicities of the corresponding homology classes in the fiber. All dots correspond to rational curves with self-intersection -2 . In $I_{n}^{*}$ we have a total of $n+5$ vertices; in particular, $I_{0}^{*}$ admits a vertex of valency four.

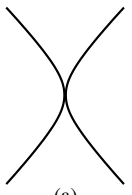

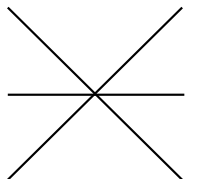

(b)

Figure 2. Singular fibers of types $I I I$ and $I V$ in elliptic fibrations. In (a) the two curves are tangent with multiplicity two, and in (b) the three curves pass through one point and intersect each other there transversely. All curves have multiplicity one and self-intersection -2 .

basic elliptic surfaces in the terminology of [9, Section 3.1.4]. This restriction is justified by the existence of the Hitchin section [12] in moduli spaces of Higgs bundles, whose construction can easily be generalized to moduli spaces of (logarithmic or irregular) singular Higgs bundles. Alternatively, it follows from [24, Section 4] that the elliptic fibrations we will encounter all originate from a pencil by blow-ups, hence all admit sections. The singular fibers in an elliptic fibration have been classified by Kodaira in [15]. From Kodaira's list of singular fibers we will only need the fibers $\widetilde{E}_{8}, \widetilde{E}_{7}, \widetilde{E}_{6}, I I, I I I, I V$ and $I_{n}^{*}(n \geq 0)$, see Figs. 1 and 2 for their description. (The fiber of type $I I$ is not given pictorially - it is the cusp fiber, which is topologically a sphere with a singular point, where the singularity can be modeled by a cone on the trefoil knot. In $\mathbb{C} P^{2}$ such a curve can be given in homogeneous coordinates $[x: y: z]$ by the equation $z y^{2}=x^{3}$.)

Various possibilities of singular fibers in an elliptic fibration on a rational elliptic surface were analyzed in detail in $[18,20,23]$. In particular, we have

Theorem 4.1 ([23, Section 6]). Suppose that $\pi: X \rightarrow \mathbb{C} P^{1}$ is an elliptic fibration on a rational elliptic surface $X$. Assume furthermore that $\pi$ admits a singular fiber of type $I_{n}^{*}$. Then $n \leq 4$. Furthermore, if $\pi$ has exactly two singular fibers, and one of them is of type $I_{n}^{*}$, then $n=0$ and both fibers are of type $I_{0}^{*}$.

Corollary 4.2. There is no elliptic fibration on the rational elliptic surface with exactly two singular fibers, one of which is $I_{n}^{*}$ with $n>0$.

We analyze the fibration with two $I_{0}^{*}$ fibers a little further. We can construct such a fibration by considering the following pencils.

Example 4.3. Consider the Hirzebruch surface $\mathbb{F}_{2}$ with infinity section of square $(-2)$ and section of square 2 . Let $C_{0}$ be the curve given by a section with multiplicity 2 . Consider $C_{\infty}$ as the union of the section at infinity with multiplicity 2 together with 4 distinct fibers of the 

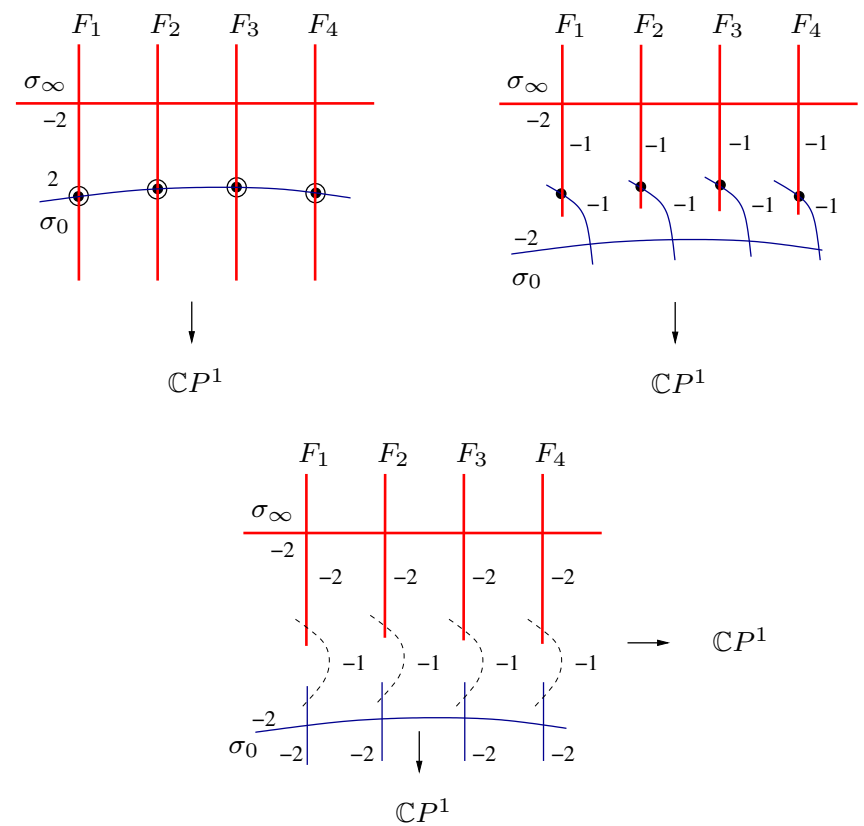

Figure 3. On the left the Hirzebruch surface (with the ruling), together with the curves $C_{\infty}=\sigma_{\infty} \cup$ $F_{1} \cup F_{2} \cup F_{3} \cup F_{4}$ and $C_{0}=\sigma_{0}$ is depicted. The concentric circles indicate double blow-ups - after one blow-up at each of the four points we get the middle configuration, while four further blow-ups provide the right hand diagram, with two $I_{0}^{*}$ fibers.

ruling on $\mathbb{F}_{2}$. It is easy to see that the two curves are homologous, and indeed, can be given as zero-sets of two sections of the same holomorphic line bundle, hence there is a pencil of curves containing both. Blowing up the four basepoints (which are the intersections of the fibers with the section) twice, we get an elliptic fibration on the 8-fold blow-up of $\mathbb{F}_{2}$ (which is a rational elliptic surface), with two singular fibers, each of type $I_{0}^{*}$. For the blow-up process, see Fig. 3.

In some sense the converse of the above example also holds. Indeed, if we get a fibration by blowing up a pencil on $\mathbb{F}_{2}$ containing $C_{\infty}$, then the pencil has at least 4 basepoints, each on different fibers of the ruling on $\mathbb{F}_{2}$, hence the fibration has at least four disjoint $(-1)$-sections. In addition, these sections intersect different curves in the fiber (of type $I_{0}^{*}$ ) coming from the curve $C_{\infty}$ at infinity.

Lemma 4.4. Suppose that $\pi: X \rightarrow \mathbb{C} P^{1}$ is an elliptic fibration with two singular fibers $F_{0}, F_{1}$, both of type $I_{0}^{*}$, and the fibration admits four disjoint sections (of homological square (-1)) which intersect different leaves of $F_{0}$. Then this fibration comes from the construction of Example 4.3.

Proof. Let $G_{i}^{1}, G_{i}^{2}, G_{i}^{3}$ and $G_{i}^{4}$ denote the (-2)-curves in the fiber $F_{i}$ (with $i=0,1$ ) corresponding to the leaves of the plumbing presentation of these fibers of type $I_{0}^{*}$. Suppose furthermore that the four sections are denoted by $E_{1}, E_{2}, E_{3}$ and $E_{4}$, with the understanding that $E_{j}$ intersects $G_{0}^{j}$ (and no other $G_{0}^{k}$ ), see Fig. 4 .

We can also assume (after possibly renumbering) that $G_{1}^{1}$ is the curve among the $G_{1}^{j}$ 's intersected by the most $E_{i}$ 's. Note that each section intersects a fiber of type $I_{0}^{*}$ only in curves corresponding to leaves (since the central curve has multiplicity 2, while a section intersects the fiber exactly once (with multiplicity)).

If $G_{1}^{1}$ is intersected by a single $E_{i}$, then by our choice each $G_{1}^{j}$ is intersected by a single section, hence blowing down first the sections and then the images of the curves $G_{1}^{j}$ 's, we get the configuration of curves described in Example 4.3. 


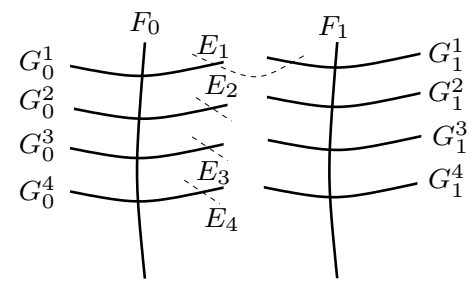

Figure 4. The two $I_{0}^{*}$ fibers and the sections $E_{i}$ are shown in the diagram. $E_{1}$ intersects $G_{1}^{1}$; the further intersections of the $E_{i}$ 's with $i>1$ provide various cases, analysed in the proof.

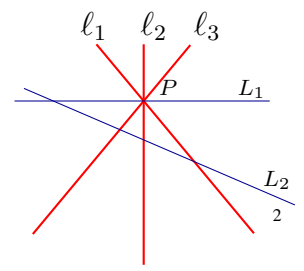

Figure 5. Curve configuration in $\mathbb{C} P^{2}$ used in Example 4.5.

Suppose now that $G_{1}^{1}$ is intersected by two $E_{i}$ 's, say by $E_{1}$ and $E_{2}$. Blow these curves and $G_{0}^{1}, G_{0}^{2}$ down. In the result the curve $G_{1}^{1}$ will be a $(+2)$-curve, hence the complement of this curve should be negative definite. On the other hand, $E_{3}$ (intersecting $G_{0}^{3}$ ) also intersects the fiber $F_{1}$, in the curve $G_{1}^{2}$ (after possible renumbering). Now blowing down $E_{3}$ and $G_{1}^{2}$, the curve $G_{0}^{3}$ will be a complex curve of self-intersection 0. Since a complex curve does not vanish in homology, it defines a nontrivial homology class in the complement of the $(+2)$-curve with zero self-intersection, providing a contradiction.

Assume now that $G_{1}^{1}$ is intersected by three sections, say $E_{1}, E_{2}$ and $E_{3}$. Blowing these and the corresponding curves from $F_{0}$ (i.e., $G_{0}^{1}, G_{0}^{2}, G_{0}^{3}$ ) down, the curve defined by $G_{1}^{1}$ will have self-intersection $(+4)$ in $\mathbb{C} P^{2} \# 3 \overline{\mathbb{C} P}^{2}$ with four curves $\left(G_{0}^{4}, G_{1}^{2}, G_{1}^{3}, G_{1}^{4}\right)$ in its complement, linearly independent in homology. This is a contradiction again.

Assume finally that all four sections intersect $G_{1}^{1}$. Blowing the sections and then the curves $G_{0}^{i}$ down, we get the image of $G_{1}^{1}$ in $\mathbb{C} P^{2} \# \overline{\mathbb{C P}}^{2}$ of self-intersection $(+6)$, with three curves in the complement, defining linearly independent homology classes, providing the last desired contradiction.

Example 4.5. In a similar manner, we can specify a pencil of curves in the complex projective plane which (after 9 blow-ups) provides an elliptic fibration with two singular fibers, both of type $I_{0}^{*}$. Indeed, let $S_{\infty}=\cup_{i=1}^{3} \ell_{i}$ be the union of three lines $\ell_{1}, \ell_{2}, \ell_{3}$ passing through a fixed point $P \in \mathbb{C} P^{2}$, each line with multiplicity one. Define $S_{0}$ to be the union $L_{1} \cup L_{2}$, where $L_{1}$ passes through $P$ while $L_{2}$ does not; we consider $L_{1}$ with multiplicity 1 and $L_{2}$ with multiplicity 2, see Fig. 5 . In this way both $S_{\infty}$ and $S_{0}$ are (singular) curves of degree 3 , and the resulting pencil (with four basepoints, the three intersection points $\ell_{i} \cap L_{2}$ and $P$ ) will define an elliptic fibration with exactly two $I_{0}^{*}$ fibers and no further singular fibers.

Example 4.6. A more symmetric presentation of the same fibration can be given as follows. Consider $\mathbb{C} P^{1} \times \mathbb{C} P^{1}$ (which is the Hirzebruch surface $\mathbb{F}_{0}$ ), together with the ruling $r: \mathbb{C} P^{1} \times \mathbb{C} P^{1}$ $\rightarrow \mathbb{C} P^{1}$. Choose two sections $S_{0}$ and $S_{\infty}$ of the ruling, and four distinct fibers $F_{1}, F_{2}, F_{3}, F_{4}$. Define the two curves $C_{0}, C_{\infty}$ by

$$
C_{0}=S_{0} \cup F_{1} \cup F_{2}, \quad C_{\infty}=S_{\infty} \cup F_{3} \cup F_{4},
$$




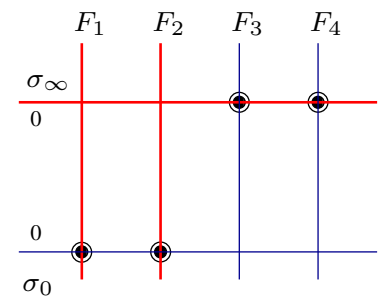

Figure 6. The symmetric curve configuration in $\mathbb{F}_{0}=\mathbb{C} P^{1} \times \mathbb{C} P^{1}$ providing a elliptic fibration with two $I_{0}^{*}$ fibers.

where each $F_{i}$ appears with multiplicity 1 , while both $S_{0}$ and $S_{\infty}$ appear with multiplicity 2 , see Fig. 6. The two curves obviously generate a pencil on $\mathbb{C} P^{1} \times \mathbb{C} P^{1}$ with four basepoints. Blowing up each basepoints twice, we get the desired elliptic fibration with two singular fibers, each of the type of $I_{0}^{*}$, originated from the curves $C_{0}$ and $C_{\infty}$.

\section{The proofs}

In this section we give the proofs of Theorems 1.1-1.6. For sake of completeness and in order to have a uniform treatment, we include and provide references to our previously obtained results whenever applicable.

\subsection{The case of one pole}

Proof of Theorems 1.1 and 1.2. These theorems are special cases of [13, Theorem 1.1] and [13, Theorem 1.3], respectively; hence we do not give any further details here.

\subsection{The case of two poles}

This case has been extensively studied in [14]. Recall that in this case we have two subcases depending on the multiplicities of the poles: these multiplicities can be (a) 2 and 2 or (b) 3 and 1.

If the two multiplicities are 2 and 2 , then the fiber at infinity is either of type $I_{2}^{*}, I_{3}^{*}$ or $I_{4}^{*}$. According to Corollary 4.2, elliptic fibrations with those singular fibers on a rational elliptic surface have at least two further singular fibers, hence they do not occur in the cases under the current investigation. (For the complete picture in this case see [14, Theorems 2.1, 2.2, 2.3], depending on whether the polar parts are twisted or untwisted. See also [14, Table 1].)

Proof of Theorems 1.3 and 1.4. Suppose now that the two multiplicities are 3 and 1. By examining [14, Theorems 2.4, 2.5, 2.6, 2.7], we see that there are two cases with single singular fibers in the Hitchin fibration, as given in the two theorems.

\subsection{The case of three poles}

Suppose now that there are three poles. In this case the fiber at infinity in the elliptic fibration is either of type $I_{1}^{*}$ (when the polar part at the pole with multiplicity 2 is untwisted (i.e., regular semi-simple)) or of type $I_{2}^{*}$ (when this polar part is twisted, i.e., has non-vanishing nilpotent part).

Proof of Theorem 1.5. According to Corollary 4.2, next to these singular fibers we cannot have a single singular fiber in any elliptic fibration on a rational elliptic surface, verifying the statement of the theorem. 


\subsection{The case of four poles}

Proof of Theorem 1.6. Consider the curve $C_{\infty} \subset \mathbb{F}_{2}$ which is the union of the section at infinity (with self-intersection $(-2)$ ) with multiplicity 2 and four fibers of the ruling on $\mathbb{F}_{2}$. We can choose coordinates on $\mathbb{C} P^{1}$ in such a way that the four fibers are over $0,1, \infty$ and $t$, where $t \in \mathbb{C}$ described the holomorphic type of the fiber at infinity.

By Lemma 4.4 the pencil containing the above curve provides an elliptic fibration with two $I_{0}^{*}$ fibers if and only if the pencil contains a section of the ruling $p: \mathbb{F}_{2} \rightarrow \mathbb{C} P^{1}$ (which is of multiplicity 2). By virtue of Proposition 3.5, in this case the Hitchin fibration on the extended moduli space $\overline{\mathcal{M}}$ of (1.1) also has a singular fiber of type $I_{0}^{*}$ for generic parabolic weights. In particular, the pencil has four basepoints, one on each of the four distinguished fibers of the ruling of $\mathbb{F}_{2}$. Suppose that the four basepoints are at $a, b, c, d$ in the fibers over the points $0,1, t, \infty \in \mathbb{C} P^{1}$.

The ruling $p: \mathbb{F}_{2} \rightarrow \mathbb{C} P^{1}$ admits a complex 3-dimensional family of sections (all disjoint from the section at infinity), which can be given by homogeneous degree- 2 polynomials on $\mathbb{C} P^{1}$. In particular, if the homogeneous coordinates on $\mathbb{C} P^{1}$ are denoted by $[u: v]$, then a section is of the form $\alpha v^{2}+\beta u v+\gamma u^{2}$. The section intersects the fiber over 0 (given by the homogeneous coordinates $[0: 1]$ ) at $\alpha$, hence we need $\alpha=a$. Similarly, over $\infty=[1: 0]$ the intersection point is $\gamma$, so we have $\gamma=d$. Finally, over the point $1=[1: 1] \in \mathbb{C} P^{1}$ we get that $\alpha+\beta+\gamma=b$, implying $\beta=b-a-d$. In conclusion, the unique section intersecting the fibers over $0,1, \infty$ in the prescribed points $a, b, d$ can be given by the equation

$$
a v^{2}+(b-a-d) u v+d u^{2} .
$$

This section takes the value $a+(b-a-d) t+d t^{2}$ at $t=[t: 1]$, providing the condition

$$
c=a+(b-a-d) t+d t^{2}
$$

for the existence of the desired section (and therefore for the other fiber to be of type $I_{0}^{*}$ ), and concluding the proof of the statement.

This argument concludes the proof of Theorems 1.1-1.6. We are left with Theorem 1.7, the proof of which will be provided in the next section.

\section{Pencils and fibrations with fiber $I_{0}^{*}$}

We devote this section to the proof of Theorem 1.7. We will describe pencils in the Hirzebruch surface $\mathbb{F}_{2}$, and sometimes (for the sake of convenience) we also provide a pencil in the complex projective plane giving rise to a fibration with the same desired properties. There is a rather simple procedure for converting pencils in $\mathbb{F}_{2}$ to pencils in $\mathbb{C} P^{2}$ (by blowing up and down); in most cases we will omit the actual steps of this procedure.

The possible combinations of singular fibers in elliptic fibrations on a rational elliptic surface have been determined in [18, 20], see also [23]. Recall the types of elliptic singular fibers listed in Section 4; there are a few further types we need to encounter in the following. $I_{1}$ denotes the nodal fiber (also called a fishtail fiber), which is a nodal elliptic curve, and topologically an immersed 2-sphere with one positive transverse double point. The fiber $I_{n}$ consists of $n$ rational curves $E_{0}, \ldots, E_{n-1}$ such that $E_{i}$ transversely intersects $E_{i-1}$ and $E_{i+1}$ in two distinct points (one each), and the indices are viewed $\bmod n$. In particular, $I_{2}$ consists of two rational curves $E_{0}$ and $E_{1}$ intersecting each other in two distinct points - as the two points converge to each other, the limit of such a sequence of singular fibers is a type $I I I$ fiber.

There are 19 distinct configurations of singular fibers on $\mathbb{C} P^{2} \# 9 \overline{\mathbb{C} P^{2}}$ containing $I_{0}^{*}$ as one of the singular fiber, which we list now (separating the singular fibers in a fibration with an 
addition sign), grouped in four groups. Below we list the fibers next to the (always existing) $I_{0}^{*}$ fiber:

$$
\begin{aligned}
& I_{0}^{*}, \quad I_{4}+2 I_{1}, \quad I V+I I, \quad I V+2 I_{1}, \quad I_{3}+I I+I_{1}, \quad I_{3}+3 I_{1} \\
& 2 I I I, \quad I I I+I_{2}+I_{1}, \quad I I I+I I+I_{1}, \quad I I I+3 I_{1} ; \\
& 3 I_{2}, \quad 2 I_{2}+2 I_{1}, \quad I_{2}+2 I I, \quad I_{2}+I I+2 I_{1}, \quad I_{2}+4 I_{1} ; \\
& 3 I I, \quad 2 I I+2 I_{1}, \quad I I+4 I_{1}, \quad 6 I_{1} \text {. }
\end{aligned}
$$

Table 1. Possible singular fiber combinations next to $I_{0}^{*}$.

In the following we give examples of pencils of curves in $\mathbb{F}_{2}$ (and sometimes in $\mathbb{C} P^{2}$ ) providing elliptic fibrations on rational elliptic surfaces containing the configurations listed above. The curves are chosen so that we do have control on the further singular fibers of the fibration. In the following we will describe pencils in $\mathbb{F}_{2}$ and in $\mathbb{C} P^{2}$ by specifying two curves (in each complex surface) and consider the pencil generated by them - and the singular fibers will then be in the fibrations we get by blowing up the base points of these pencils.

The curve $C_{\infty} \subset \mathbb{F}_{2}$ will be part of all the pencils; $C_{\infty}$ consists of the union of four fibers $F_{1}, F_{2}, F_{3}, F_{4}$ of the ruling $\mathbb{F}_{2} \rightarrow \mathbb{C} P^{1}$ (all with multiplicity 1 ) and the section at infinity of the ruling (this curve with multiplicity 2) - as it was already described in Example 4.3. Similarly, one curve in each pencil in $\mathbb{C} P^{2}$ will be the same: $S_{\infty}=\ell_{1} \cup \ell_{2} \cup \ell_{3}$ is the cubic curve we get by taking the union of three projective lines $\ell_{i}(i=1,2,3)$ (all with multiplicity 1 ) passing through a fixed point $P \in \mathbb{C} P^{2}$. The curve $S_{\infty}$ is shown by the thick red lines on the right of the figures below. (This curve already appeared in Example 4.5.)

We say that a pencil in $\mathbb{F}_{2}$ has a section if one of the curves in the pencil other than $C_{\infty}$ has more than one components - in this case each component of this curve is indeed a section of the ruling of the Hirzebruch surface $\mathbb{F}_{2}$.

In some cases we need to see more than two singular fibers in the fibration. To this end, we will apply a principle (formulated in Lemma 6.1 below) and a method of generating more curves in a pencil (described after Lemma 6.1).

Suppose that the pencil is given by the two curves $C_{0}$ and $C_{\infty}$ in $\mathbb{F}_{2}$ (or $S_{0}$ and $S_{\infty}$ in $\mathbb{C} P^{2}$ ), intersecting each other in points $P_{1}, \ldots, P_{k}$ with multiplicities $n_{1}, \ldots, n_{k}$, satisfying $\sum_{i=1}^{k} n_{i}=8$ in $\mathbb{F}_{2}$ and $\sum_{i=1}^{k} n_{i}=9$ in $\mathbb{C} P^{2}$. (In case $n_{i}>1$, not only $P_{i}$ is fixed, but also the higher order tangencies are part of the given data, for example by specifying the points in the exceptional divisor of the blow-up the strict transforms of the curves pass through.) We mentioned that the elements of matrices in equations (2.9) and (2.10) encode the base locus of the pencil, hence $P_{1}, \ldots, P_{k}$ are determined by these data. Next, we will construct some examples where we choose these parameters $P_{1}, \ldots, P_{k}$ appropriately on four distinguished fibers of the ruling, so that all combinations of further singular fibers appear. It is easy to see that these chosen parameters can be transformed into $a_{ \pm}, \ldots, d_{ \pm}, a, \ldots, d$. (For convenience, from now on we will not insist on having the distinguished fibers over the points $0,1, \infty$ - the application of a simple Möbius transformation would provide this extra feature.)

Suppose now that $C$ (or $S$ ) is a complex curve in $\mathbb{F}_{2}$ (or in $\mathbb{C} P^{2}$, respectively) which is homologous to $C_{0}$ and $C_{\infty}$ (or $S_{0}$ and $S_{\infty}$ ) and passes through $P_{1}, \ldots, P_{k}$, intersecting $C_{0}$ (or $S_{0}$ ) in those points with multiplicities $n_{1}, \ldots, n_{k}$ (and with the required higher order tangencies in case $\left.n_{i}>1\right)$.

Lemma 6.1. Under the above circumstances $C($ or $S)$ is in the pencil in $\mathbb{F}_{2}$ generated by $C_{0}$ and $C_{\infty}\left(\right.$ or $S_{0}$ and $S_{\infty}$ in case we work in $\left.\mathbb{C} P^{2}\right)$.

Proof. Take any point $P \in C \backslash\left\{P_{1}, \ldots, P_{k}\right\}$. There exists a unique $t \in \mathbb{C} P^{1}$ such that $C_{t}$ passes through $P$. We then see that $C$ intersects $C_{t}$ with multiplicity $>\sum_{i=1}^{k} n_{i}$, which is by assumption the self-intersection number of $C$. It then follows that $C=C_{t}$. 

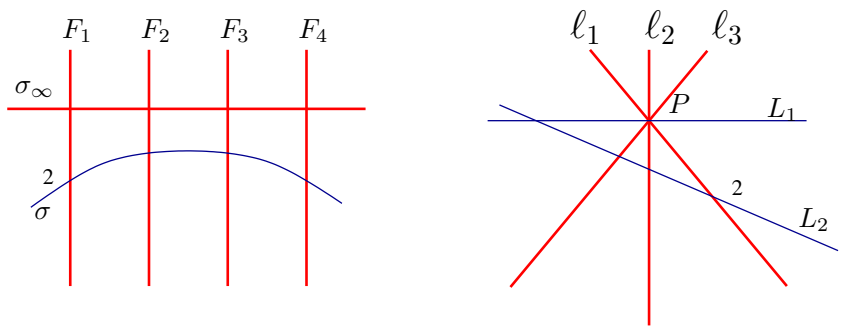

Figure 7. The diagram on the left shows two curves in $\mathbb{F}_{2}$, one of them is the curve $C_{\infty}$, while the other one is a section of the ruling with multiplicity two (denoted by $\sigma$ ). Thick (red) lines provide one of the curves, while the thin (blue) segment defines the other curve. The fibration defined by the pencil contains two $I_{0}^{*}$ fibers. The two degree- 3 curves in $\mathbb{C} P^{2}$ on the right generate a pencil which gives rise to a fibration admitting two $I_{0}^{*}$ fibers.

We can construct curves in $\mathbb{F}_{2}$ disjoint from the section $\sigma_{\infty}$ at infinity and intersecting the fiber of the ruling twice (possibly once, with multiplicity two) in the following way. Since the curve is disjoint from $\sigma_{\infty}$, it is a double section of the bundle $\mathcal{O}(2) \rightarrow \mathbb{C} P^{1}$. Such double sections can be given by sections of $\mathcal{O}(4) \rightarrow \mathbb{C} P^{1}$ : for a given section $\sigma$ of $\mathcal{O}(4)$, use the identification $\mathcal{O}(2) \otimes \mathcal{O}(2) \cong \mathcal{O}(4)$ and get a double section of $\mathcal{O}(2)$ : over $P \in \mathbb{C} P^{1}$ take those points $\zeta \in \mathcal{O}(2)$ which satisfy $\zeta \otimes \zeta=\sigma_{P}$, see (2.3) with the notational change of denoting $G_{\theta}$ by $\sigma$. There are two such points $\left(\zeta\right.$ and $-\zeta$ ) if $\sigma_{P} \neq 0$ and there is a single one if $\sigma_{P}=0$. (Indeed, this is the classical construction of double branched covers.) In turn, sections of $\mathcal{O}(4) \rightarrow \mathbb{C} P^{1}$ can be given by homogeneous degree- 4 polynomials in the homogeneous coordinates $[u: v]$ on $\mathbb{C} P^{1}$.

Here are some instructive examples of this phenomenon:

- The polynomial $u^{4}$ (as a section of $\mathcal{O}(4)$ ) provides two sections in $\mathcal{O}(2)$ which are tangent to each other over the point $[0: 1] \in \mathbb{C} P^{1}$.

- The polynomial $u^{2} v^{2}$ gives rise to two sections of $\mathcal{O}(2)$, intersecting each other transversally in two points over $[0: 1]$ and $[1: 0] \in \mathbb{C} P^{1}$.

- The polynomial $u^{2} v(u+v)$ defines a double section in $\mathcal{O}(2)$ which has a node over $[0: 1] \in$ $\mathbb{C} P^{1}$, and is tangent to the fibers of the ruling at $[1: 0]$ and $[-1: 1] \in \mathbb{C} P^{1}$.

- The polynomial $u^{3} v$ will define a cuspidal curve which is a double section in $\mathbb{F}_{2}$ - the cusp point is over $[0: 1]$ and the fiber over $[1: 0]$ is tangent to the cuspidal curve.

We will start our examples with configurations admitting sections.

Example 6.2 (fibration with another $I_{0}^{*}$ ). If $C_{0}$ is a section of the ruling $\mathbb{F}_{2} \rightarrow \mathbb{C} P^{1}$ with square 2 and we take it with multiplicity 2 , the pencil generated by $\left(C_{0}, C_{\infty}\right)$ provides a fibration with two singular fibers, both of type $I_{0}^{*}$, cf. Fig. 7. In a similar fashion, by taking two lines $L_{1}, L_{2} \subset \mathbb{C} P^{2}$ such that $L_{1}$ passes through the fixed point $P$ while $L_{2}$ avoids it, and defining $S_{0}$ as the union of $L_{1}$ and $L_{2}$ (with $L_{1}$ of multiplicity 1 and $L_{2}$ of multiplicity 2) we get a pencil on $\mathbb{C} P^{2}$ induced by $\left(S_{0}, S_{\infty}\right)$ so that the resulting elliptic fibration has two $I_{0}^{*}$ fibers (cf. Example 4.5 and Fig. 7 ).

Example 6.3 (fibration with an $I_{4}$ fiber). A fibration with an $I_{4}$ fiber (next to the $I_{0}^{*}$ coming from $C_{\infty}$ ) can be given in $\mathbb{F}_{2}$ by considering two sections $\sigma_{1}, \sigma_{2}$ of the ruling (each with multiplicity 1) intersecting each other once on $F_{1}$ and once on $F_{4}$, as shown by the left diagram of Fig. 8. A similar pencil in $\mathbb{C} P^{2}$ can be given by taking $S_{0}=L_{1} \cup L_{2} \cup L_{3}$, where $L_{i}$ are three distinct lines, $L_{1}$ passes through $P$, while $L_{2}, L_{3}$ do not, and moreover the intersection point $L_{2} \cap L_{3}$ is on one of the $\ell_{i}$; the configuration is shown by the right diagram of Fig. 8. Note that by the classification of possible singular fibers, it follows that next to the $I_{0}^{*}$ and $I_{4}$ fibers there is a single possibility for other singular fibers: these must be two fishtail $\left(I_{1}\right)$ fibers. 

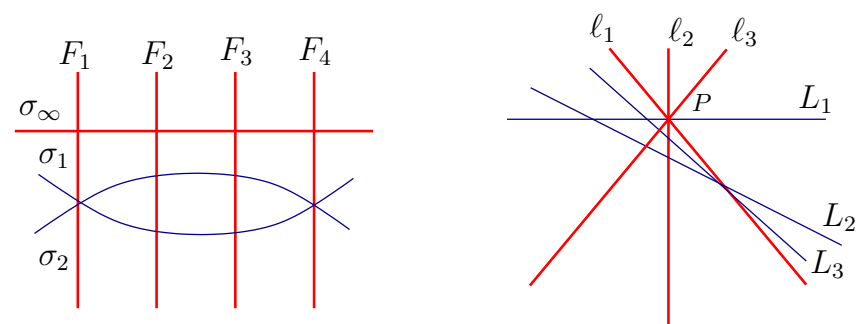

Figure 8. The diagram on the left shows two sections in $\mathbb{F}_{2}$ intersecting each other in two points of $C_{\infty}$. (The sections are denoted by $\sigma_{1}$ and $\sigma_{2}$.) Once again, thick (red) lines provide one of the curves, while the thin (blue) segments define the other curve. The pencil generated by these curves provides a fibration with an $I_{0}^{*}$ fiber and an $I_{4}$ fiber. The two degree-3 curves in $\mathbb{C} P^{2}$ on the right diagram generate a pencil which gives rise to a fibration admitting an $I_{0}^{*}$ fiber and an $I_{4}$ fiber.
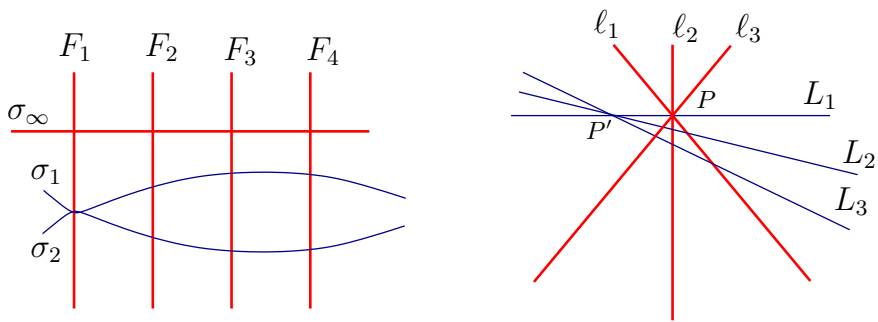

Figure 9. The diagram depicts two pencils (one in $\mathbb{F}_{2}$ and the other one in $\mathbb{C} P^{2}$ ) giving fibrations with an $I_{0}^{*}$ fiber and a type $I V$ fiber. The conventions are the same as earlier.

Example 6.4 (fibration with a type $I V$ fiber). To get a fibration with a fiber of type $I V$ next to $I_{0}^{*}$, take two sections in $\mathbb{F}_{2}$, which are now tangent to each other at a point of $F_{1}$, see the left diagram of Fig. 9. In $\mathbb{C} P^{2}$ consider $S_{0}$ to be three distinct lines $L_{1}, L_{2}, L_{3}$ again, so that $L_{1}$ passes through $P$, while all three lines pass through $P^{\prime} \in \mathbb{C} P^{2}$ which is distinct from $P$, see the right diagram of Fig. 9.

In order to get a pencil generating a fibration with an $I_{0}^{*}$, a type $I V$ and a type $I I$ fiber, consider the sections $u^{4}$ and $u v^{3}$ in $\mathcal{O}(4)$, giving rise to two double sections in $\mathbb{F}_{2}$. Let $F_{1}$, $F_{2}, F_{3}, F_{4}$ be chosen as follows: $F_{1}$ is over $[0: 1]$ (where the two sections are tangent to each other in one of the double section), and the other fibers are over $\left[u_{i}: 1\right]$, where $u_{i}^{3}=1$. The corresponding fibers pass through the intersections of the sections of $\mathcal{O}(4)$, hence each such fiber passes through two basepoints in the pencil in $\mathbb{F}_{2}$, therefore both curves are in the pencil.

In a similar manner, when starting with the sections $u^{4}$ and $u v^{2}(u+v)$, we get a pencil giving a fibration with an $I_{0}^{*}$ fiber, a type $I V$ fiber and an $I_{1}$ fiber, hence (again by the classification) there is a single further singular fiber which is an $I_{1}$. (The fibers are over the points $\left[u_{i}: 1\right]$ where $u_{i}$ solves $u^{3}=u+1$.) These two examples exhaust the cases having an $I_{0}^{*}$ fiber and a type $I V$ fiber next to it.

Example 6.5 (fibration with an $I_{3}$ fiber). Consider now two sections $\sigma_{1}, \sigma_{2}$ of the ruling on $\mathbb{F}_{2}$ intersecting each other in $F_{1}$, and in a further point which is not on the chosen fibers $F_{i}$ $(i=2,3,4)$. Taking this curve as $C_{0}$, the resulting pencil will have an $I_{3}$ fiber next to the $I_{0}^{*}$ fiber. In $\mathbb{C} P^{2}$ we consider $S_{0}$ which is again a union of three lines $L_{1}, L_{2}, L_{3}$, with $L_{1}$ passing through $P$ and $L_{2}, L_{3}$ intersecting each other in a generic point $P^{\prime}$ (which is not on $L_{1}$ and on any $\left.\ell_{i}\right)$; see Fig. 10. Fibrations admitting an $I_{0}^{*}$ and an $I_{3}$ fiber on the rational elliptic surface have further singular fibers; the possibilities are: either a cusp and a fishtail, or three fishtails. The second case is the generic one. Let us see how to construct such a fibration explicitly. For 

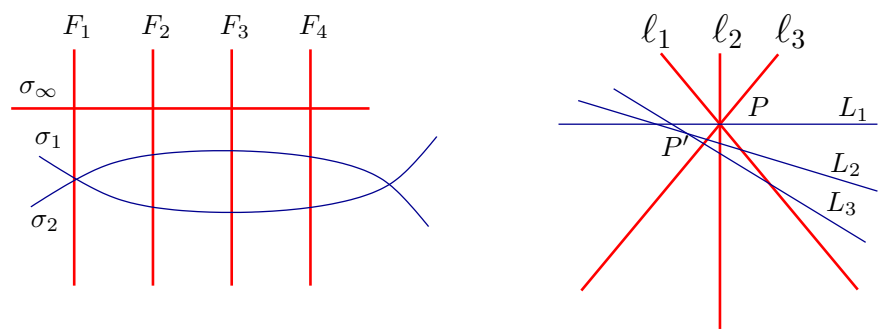

Figure 10. The diagram depicts two pencils (one in $\mathbb{F}_{2}$ and the other one in $\mathbb{C} P^{2}$ ) giving fibrations with an $I_{0}^{*}$ fiber and an $I_{3}$ fiber. The conventions are the same as earlier.

this purpose, we take

$$
\sigma_{1}=(u+v)^{2} v(u-v), \quad \sigma_{2}=u^{2} v^{2} .
$$

Then $\sigma_{1}$ is not a complete square, but has two equal zeroes, so the equation $\zeta \otimes \zeta=\sigma_{1}$ defines a double section with a nodal point over $[u: v]=[-1: 1]$, giving rise to a fiber of type $I_{1}$. On the other hand, $\sigma_{2}$ is a complete square with zeroes at $[u: v]=[0: 1]$ and $[u: v]=[1: 0]$. The basepoints are determined by the equation

$$
(u+v)^{2} v(u-v)=u^{2} v^{2},
$$

which has roots $[u: v]=[1: 0]$ and $[u: v]=\left[u_{i}: 1\right]$ where $u_{1}, u_{2}, u_{3}$ are the roots of

$$
(u+1)^{2}(u-1)-u^{2}=0 .
$$

As one of the roots $[1: 0]$ of $\sigma_{2}$ is at the same time a basepoint while its other root $[0: 1]$ is not, $\sigma_{2}$ gives rise to a fiber of type $I_{3}$. Now, let the parameter of the pencil be denoted $\lambda=\left[\lambda_{1}: \lambda_{2}\right] \in \mathbb{C} P^{1}$. Then, the curve corresponding to $\lambda$ in the pencil has equation

$$
\left(\lambda_{1}+\lambda_{2}\right) \zeta \otimes \zeta=\sigma_{\lambda}
$$

where

$$
\sigma_{\lambda}=\lambda_{1} \sigma_{1}+\lambda_{2} \sigma_{2}
$$

The parameter value $\lambda=[-1: 1]$ corresponds to a degenerate curve consisting of the fibers containing the basepoints (and the section at infinity in $\mathbb{F}_{2}$ with multiplicity 2), and gives rise to the fiber of type $I_{0}^{*}$. From now on we assume that $\lambda \neq[-1: 1]$, and the equation of the corresponding curve can be written as

$$
\zeta \otimes \zeta=\frac{\sigma_{\lambda}}{\lambda_{1}+\lambda_{2}}
$$

Expressing the right hand side of this equation using equation (6.1), and taking $v=1$ we get

$$
\lambda_{1} u^{3}+\left(\lambda_{1}+\lambda_{2}\right) u^{2}-\lambda_{1} u-\lambda_{1} .
$$

The discriminant of this polynomial is given by

$$
\lambda_{1} \lambda_{2} \frac{32 \lambda_{1}^{2}+13 \lambda_{1} \lambda_{2}+4 \lambda_{2}^{2}}{\left(\lambda_{1}+\lambda_{2}\right)^{4}}
$$



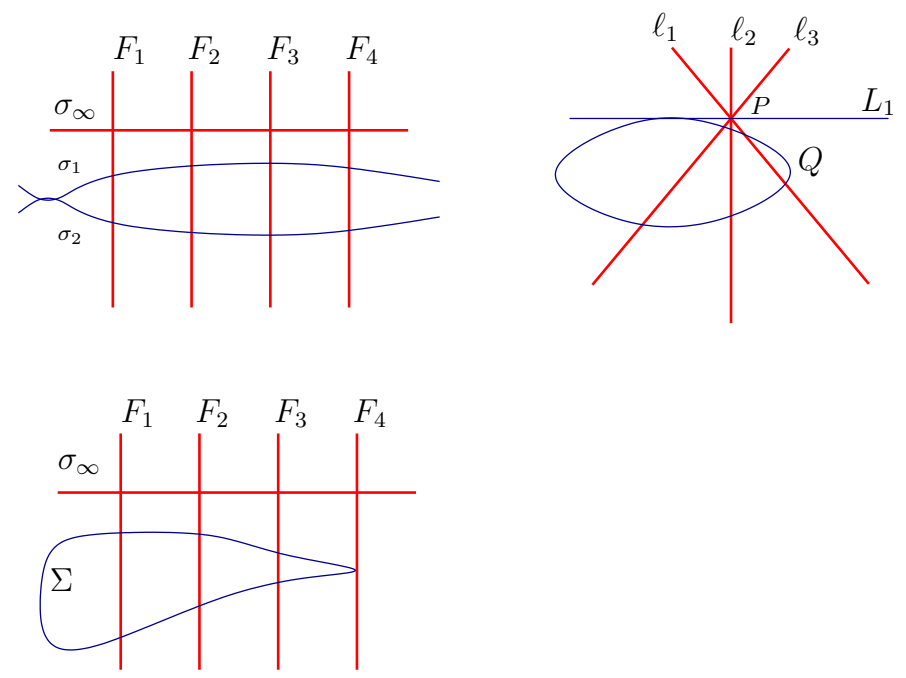

Figure 11. Continuing with the same conventions as above, the diagrams show pencils defining fibrations with an $I_{0}^{*}$ fiber and a type $I I I$ fiber. In this case we describe two pencils in $\mathbb{F}_{2}$, one having eight, the other one seven basepoints.

The parameter values $\lambda_{1}=0$ and $\lambda_{2}=0$ define $\sigma_{2}$ and $\sigma_{1}$ respectively, hence we have already treated them. (Remember that $\lambda_{1}, \lambda_{2}$ may not simultaneously vanish.) Consider the roots $\lambda^{+}=\left[\lambda_{1}^{+}: \lambda_{2}^{+}\right], \lambda^{-}=\left[\lambda_{1}^{-}: \lambda_{2}^{-}\right] \in \mathbb{C} P^{1}$ of

$$
32 \lambda_{1}^{2}+13 \lambda_{1} \lambda_{2}+4 \lambda_{2}^{2}
$$

a straightforward check shows that $\lambda^{+} \neq \lambda^{-}$and that

$$
\lambda^{+}, \lambda^{-} \in \mathbb{C} \backslash\{[0: 1],[-1: 1]\} \subset \mathbb{C} P^{1} .
$$

Then, $\sigma_{\lambda^{ \pm}}$both have a double zero, therefore each of the corresponding curves has at least one singular point. Moreover, these singular curves are different from the ones we found earlier. By the classification, the corresponding curves in the fibration are both of type $I_{1}$, and we are done.

Now, let us turn our attention to finding an example of the non-generic case, i.e., a cuspidal curve beside $I_{0}^{*}$ and $I_{3}$. Using our earlier method of generating double sections from homogeneous degree-4 polynomials, we proceed as follows. Take the polynomials $u^{2} v^{2}$ and $u(u+v)^{3}$, and consider the fibers $F_{1}, \ldots, F_{4}$ over the points $\left[u_{i}: 1\right] \in \mathbb{C} P^{1}$ for which $u^{2}=u(u+1)^{3}$ holds. These are the fibers passing through the intersection points of the sections in $\mathcal{O}(4)$, hence of pairs of basepoints in $\mathbb{F}_{2}$. The second curve is a cuspidal curve, hence the resulting fibration will have an $I_{0}^{*}$ fiber, together with an $I_{3}$ fiber and a cusp (and a further $I_{1}$ fiber, as dictated by the classification).

Example 6.6 (fibration with $I I I)$. The curve $C_{0}$ in $\mathbb{F}_{2}$ is a union of two sections, which are tangent to each other in a point not on the chosen fibers $F_{i}(i=1,2,3,4)$. (Alternatively, if we get a fibration with a double-section which has a cusp point on the fiber $F_{4}$ - as shown in Fig. 11 - we get a fibration with an $I_{0}^{*}$ fiber and a type $I I I$ fiber.) The curve $S_{0}$ in the pencil in $\mathbb{C} P^{2}$ now consists of two curves, a line $L_{1}$ passing through $P$ and a quadric $Q$ which does not pass through $P$ and it is tangent to $L_{1}$, as shown by the right diagram of Fig. 11. There are four possibilities of the further fibers (listed in the second row of Table 1): it can be (a) another type $I I I$ fiber, (b) $I_{2}$ and $I_{1}$, (c) $I I$ and $I_{1}$ and finally (d) three $I_{1}$ 's.

By taking the degree- 4 polynomials $u^{3} v$ and $u v^{3}$ and the fibers over $[0: 1],[ \pm 1: 1],[1: 0]$ $\in \mathbb{C} P^{1}$, the pencil will give rise to a fibration with an $I_{0}^{*}$ fiber and two type $I I I$ fibers. Similarly, 

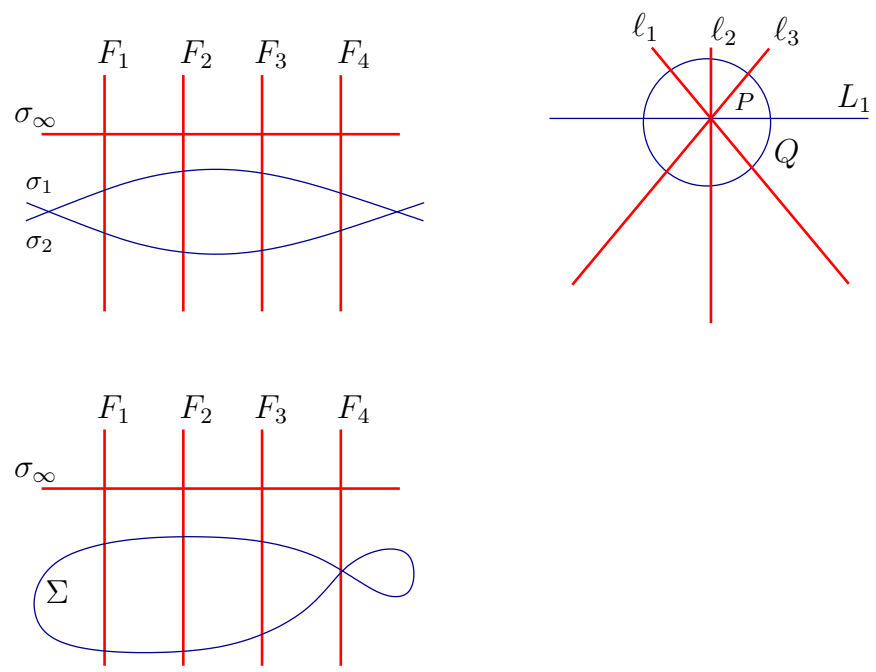

Figure 12. The pencils give rise to fibrations with an $I_{0}^{*}$ fiber and an $I_{2}$ fiber.

by taking $u^{4}$ and $(u+v) v^{3}$ and the fibers over $\left[u_{i}: 1\right]$ with $u_{i}$ solving $u^{4}=(u+1)$, we get a pencil giving a fibration with an $I_{0}^{*}$, a type $I I I$ and a cusp (type $I I$ ) fibers, and hence necessarily a further $I_{1}$ fiber. In a similar manner, starting with $u^{3} v$ and $u v^{2}(u+v)$, and taking the fibers over $[0: 1],[1: 0] \in \mathbb{C} P^{1}$ and $\left[u_{i}: 1\right] \in \mathbb{C} P^{1}$ with $u_{i}$ solving $u^{2}=u+1$, we get a pencil giving a fibration with an $I_{0}^{*}$ fiber, a type $I I I$ and an $I_{2}$ fibers (and therefore a further $I_{1}$ ). In the generic situation we have three $I_{1}$ fibers next to $I_{0}^{*}$ and $I I I$. This case can be shown to exist by the same method as the generic case in Example 6.5 has been handled. Specifically, setting $\sigma_{1}=u^{4}$ (giving rise to a curve of type $I I I$ with singularity over $\left.[0: 1]\right)$ and $\sigma_{2}=(u-v)^{2} v(u+v)$ (giving rise to a curve of type $I_{1}$ with singularity over $[1: 1]$ ), the generic curve in the pencil again reads as

$$
\sigma_{\lambda}=\lambda_{1} \sigma_{1}+\lambda_{2} \sigma_{2}
$$

A computation shows that the discriminant of $\sigma_{\lambda}$ is given by

$$
\Delta=\lambda_{1} \lambda_{2}^{3}\left(256 \lambda_{1}^{2}-107 \lambda_{1} \lambda_{2}-32 \lambda_{2}^{2}\right) .
$$

The quadratic term in the parentheses admits two distinct roots in $\mathbb{C}^{\times}$, so there exist two values $\lambda^{ \pm}=\left[\lambda_{1}^{ \pm}: \lambda_{2}^{ \pm}\right]$such that $\sigma_{\lambda^{ \pm}}$is singular. By the classification, in the fibration these singular curves must be of type $I_{1}$. Hence, together with $\left[\lambda_{1}: \lambda_{2}\right]=[0: 1]$ and $\left[\lambda_{1}: \lambda_{2}\right]=[1: 0]$ we have a curve of type $I I I$ and three curves of type $I_{1}$.

Example 6.7 (fibration with an $I_{2}$ fiber). To get a fibration with an $I_{2}$ fiber, we consider two sections $\sigma_{1}, \sigma_{2}$ intersecting each other in two distinct points, none of them on the chosen fibers $F_{i}(i=1,2,3,4)$. Alternatively, we can choose a nodal curve with the node on the fiber $F_{4}$, shown on Fig. 12. To get such a fibration from a pencil in $\mathbb{C} P^{2}$, take $L_{1}$ as earlier, together with a quadric, which does not pass through $P$ and it is transverse to $L_{1}$, see Fig. 12 .

There are five cases for the further singular fibers, as shown in the third row of Table 1: next to $I_{0}^{*}$ and $I_{2}$ we can have: (a) two further $I_{2}$, (b) an $I_{2}$ and two $I_{1}$, (c) two type $I I$, (d) a type $I I$ and two $I_{1}$ and finally (e) four $I_{1}$. We will discuss these cases below.

Example 6.8 (three $I_{2}$ fibers). Consider an elliptic pencil on $\mathbb{F}_{2}$ whose eight basepoints are all distinct, pairwise lying on the fibers over $0,1, t, \infty$ of the ruling. Assume in addition that there exists a section $\sigma_{1}$ of $\mathbb{F}_{2}$ passing through four of these basepoints and a section $\sigma_{2}$ passing through the other four basepoints (see the upper left diagram of Fig. 12). 
If there exist two sections $\sigma_{1}, \sigma_{2}$ satisfying the above conditions, then in the corresponding fibration they give rise to a curve of type $I_{2}$. In order to find a fibration with three $I_{2}$ fibers, it is thus sufficient to find values $a_{ \pm}, b_{ \pm}, c_{ \pm}, d_{ \pm}$such that there exist simultaneously

- a pair of sections $\sigma_{1}, \sigma_{2}$ such that $\sigma_{1}$ passes through $a_{+}, b_{+}, c_{+}, d_{+}$and $\sigma_{2}$ passes through $a_{-}, b_{-}, c_{-}, d_{-}$;

- a pair of sections $\sigma_{3}, \sigma_{4}$ such that $\sigma_{3}$ passes through $a_{-}, b_{+}, c_{-}, d_{+}$and $\sigma_{4}$ passes through $a_{+}, b_{-}, c_{+}, d_{-}$;

- a pair of sections $\sigma_{5}, \sigma_{6}$ such that $\sigma_{5}$ passes through $a_{+}, b_{+}, c_{-}, d_{-}$and $\sigma_{6}$ passes through $a_{-}, b_{-}, c_{+}, d_{+}$.

Each of these pairs of sections then give rise to different curves of type $I_{2}$ in the fibration coming from the pencil defined by the corresponding set of basepoints, and by an Euler characteristic computation this fibration has no other singular fibers.

A necessary and sufficient condition for the existence of such a pair of sections can be found as follows. In the trivialization of $K_{\mathbb{C} P^{1}}(D)$ over $U$ discussed in $(2.5)$ for $1 \leq j \leq 2$ the section $\sigma_{j}$ of $\mathbb{F}_{2}$ is of the form

$$
\sigma_{j}(u)=\alpha_{j} u^{2}+\beta_{j} u+\gamma_{j}
$$

for some $\alpha_{j}, \beta_{j}, \gamma_{j} \in \mathbb{C}$. For simplicity assume that $t=-1$. Then, $\sigma_{1}$ passes through the basepoints if and only if the following equations hold

$$
\begin{aligned}
& \gamma_{1}=a_{+}, \\
& \alpha_{1}+\beta_{1}+\gamma_{1}=b_{+}, \\
& \alpha_{1}-\beta_{1}+\gamma_{1}=c_{+}, \\
& \alpha_{1}=d_{+},
\end{aligned}
$$

where $a_{ \pm}, b_{ \pm}, c_{ \pm}$are the $w$-coordinates of the basepoints over $0,1, t=-1$ respectively and $d_{ \pm}$ are the $z$ coordinates over $\infty$. This linear system in three variables $\alpha_{1}, \beta_{1}, \gamma_{1}$ has a solution if and only if

$$
2\left(a_{+}+d_{+}\right)=b_{+}+c_{+}
$$

Similarly, the existence of $\sigma_{2}$ is equivalent to

$$
2\left(a_{-}+d_{-}\right)=b_{-}+c_{-}
$$

We may repeat the above argument to find conditions for the existence of $\sigma_{3}, \sigma_{4}, \sigma_{5}, \sigma_{6}$. These conditions, together with the ones found above for $\sigma_{1}, \sigma_{2}$, form a system of 6 homogeneous linear equations in the 8 variables $a_{ \pm}, b_{ \pm}, c_{ \pm}, d_{ \pm}$. Completing this system with the degree formula

$$
a_{+}+b_{+}+c_{+}+d_{+}+a_{-}+b_{-}+c_{-}+d_{-}=0
$$

results in a system of 7 homogeneous linear equations in 8 variables. In view of the assumption that the basepoints are all distinct, in order to get a fibration with three $I_{2}$ fibers we merely need to show that the above linear system admits generic solutions, namely solutions such that

$$
a_{+} \neq a_{-}, \quad b_{+} \neq b_{-}, \quad c_{+} \neq c_{-}, \quad d_{+} \neq d_{-} .
$$




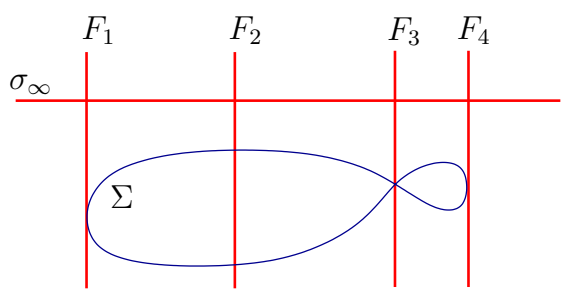

Figure 13. A pencil which gives rise to fibrations with an $I_{0}^{*}$ fiber and three $I_{2}$ fibers.

Now, the coefficient matrix with respect to the variables $a_{+}, a_{-}, \ldots, d_{+}, d_{-}$of the system of equations governing the existence of $\sigma_{1}, \ldots, \sigma_{6}$ complemented by the degree formula (6.2) reads as

$$
\left(\begin{array}{cccccccc}
2 & 0 & -1 & 0 & -1 & 0 & 2 & 0 \\
0 & 2 & 0 & -1 & 0 & -1 & 0 & 2 \\
0 & 2 & -1 & 0 & 0 & -1 & 2 & 0 \\
2 & 0 & 0 & -1 & -1 & 0 & 0 & 2 \\
2 & 0 & -1 & 0 & 0 & -1 & 0 & 2 \\
0 & 2 & 0 & -1 & -1 & 0 & 2 & 0 \\
1 & 1 & 1 & 1 & 1 & 1 & 1 & 1
\end{array}\right)
$$

One numerically checks that this matrix is of rank 5 , hence it admits a three-dimensional family of solutions. As a matter of fact, the last row of the coefficient matrix is a linear combination of the first six rows, and the corresponding last entry in the extended matrix of the linear system of equations is 0 , i.e., the degree formula automatically holds once there exists three fibers of type $I_{2}$ in the given configuration; consequently, the degree formula does not effect the solvability of the system. In addition, one sees that $c_{-}, d_{+}, d_{-}$may be chosen as parameters and the other variables may be expressed in terms of these as

$$
\begin{aligned}
& a_{+}=-d_{-}, \quad a_{-}=-d_{+}, \quad b_{+}=-c_{-}, \quad b_{-}=2 d_{-}-2 d_{+}-c_{-}, \\
& c_{+}=-2 d_{-}+2 d_{+}+c_{-} .
\end{aligned}
$$

It is therefore sufficient to choose any $d_{-} \neq d_{+}$to get solutions satisfying (6.3). Finally, we note that a similar attempt to find four $I_{2}$ fibers fails because the corresponding linear system has two free parameters $d_{+}, d_{-}$and the other variables can be expressed as

$$
a_{+}=-d_{-}, \quad a_{-}=-d_{-}, \quad \ldots
$$

In particular, there exist no solution satisfying (6.3).

Example 6.9 (three $I_{2}$ fibers). Another, less explicit construction of the same type of fibration can be given as follows: consider a nodal double section of $\mathbb{F}_{2}$ with the property that the node is on $F_{3}$ while $F_{1}$ and $F_{4}$ are tangent to the nodal double section, see Fig. 13. (It is easy to see from the Hurwitz-formula that a nodal double section can have at most two tangent fibers.) In this case an $I_{2}$ fiber is originated from the blow-up of the node of the chosen double section. On the other hand, when we blow up the basepoints where our chosen double section is tangent to $F_{1}$ and $F_{4}$, we see that disjoint rational $(-2)$-curves appear in fibers, hence by the classification the further fibers should be two $I_{2}$ fibers. (Indeed, the pencil contains two further nodal curves, their nodes are on $F_{1}$ and $F_{4}$ and are tangent to two chosen fibers.)

If only $F_{1}$ is tangent to our chosen curve (see Fig. 14), then the fibration (next to $I_{0}^{*}$ and $I_{2}$ ) has either a further $I_{2}$ (and also two $I_{1}$ ) or a type $I I I$ (and a further $I_{1}$ ). The outcome depends on the fact whether the curve with singularity on $F_{1}$ is nodal (giving a further $I_{2}$ ) or cuspidal (providing a type $I I I$ fiber). Clearly the first is the generic case among these examples. In the next examples we will give constructions of these cases. 


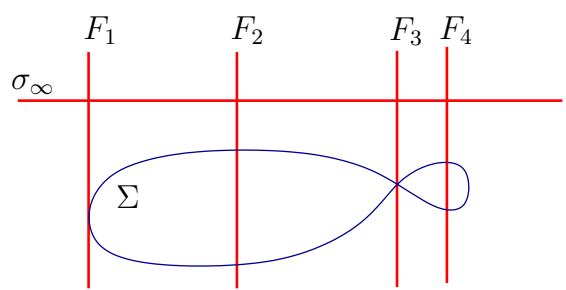

Figure 14. A pencil which gives rise to fibrations with an $I_{0}^{*}$ fiber, an $I_{2}$ fiber and further $I_{2}$ or type $I I I$ fibers.

Example 6.10 (two $I_{2}$ fibers). By choosing appropriate homogeneous polynomials, we can arrange that we have two $I_{2}$ fibers (and two further $I_{1}$ fibers). Indeed, by taking $u^{2} v(u+v)$ and $u v^{2}(u+2 v)$, for the first curve we have a node and a tangency (for the second a tangency and a node) at the fibers over $[0: 1]$ and $[1: 0]$, and by choosing the fibers over $\left[u_{i}: 1\right]$ with $u_{i}$ solving the equation $u(u+1)=u+2$, we get the desired pencil and hence fibration. Indeed, next to $I_{0}^{*}$ and the two $I_{2}$ fibers, no further fiber can contain a (-2)-curve (since the pencil cannot contain sections, and no further singular point can be on the fibers of the ruling).

A more specific construction may be given along the lines of Example 6.5, choosing $\sigma_{1}=$ $(u-v)^{2}(u+2 v)^{2}, \sigma_{2}=u^{2} v^{2}$, with the corresponding discriminant

$$
\Delta=64 \lambda_{1}^{2} \lambda_{2}^{2}\left(81 \lambda_{1}^{2}-14 \lambda_{1} \lambda_{2}+\lambda_{2}^{2}\right)
$$

admitting two distinct roots (other than at $\left[\lambda_{1}: \lambda_{2}\right]=[0: 1]$ and $[1: 0]$ ).

Example 6.11 (one $I_{2}$ fiber). A fibration with an $I_{2}$ and a cusp fiber $I I$ (next to the $I_{0}^{*}$ fiber) and by classification having either a further $I I$ or two further $I_{1}$ fibers) can be given by taking the homogeneous degree- 4 polynomials $u^{2} v^{2}$ and $(u+v)(u+x v)^{3}$ with parameter $x \neq 0,1$ (the first giving an $I_{2}$ fiber, the second a cusp curve with cusp over $\left.[-x: 1]\right)$, where the fibers of the ruling $\mathbb{F}_{2} \rightarrow \mathbb{C} P^{1}$ are chosen over $[0: 1]$ and over $\left[u_{i}: 1\right]$ with $u_{i}$ solving $u^{2}=(u+1)(u+x)^{3}$. The two cases for the additional fibers can both occur. In the pencil generated by the above two singular curves, the discriminant is

$$
\begin{aligned}
& -\lambda_{1}^{2} \lambda_{2}^{2} x^{3}\left(-16 \lambda_{1}^{2}+4 \lambda_{1} \lambda_{2}+27 \lambda_{2}^{2} x^{5}-108 \lambda_{2}^{2} x^{4}+162 \lambda_{2}^{2} x^{3}+4 \lambda_{1} \lambda_{2} x^{3}-108 \lambda_{2}^{2} x^{2}\right. \\
& \left.\quad-132 \lambda_{1} \lambda_{2} x^{2}+27 \lambda_{2}^{2} x-132 \lambda_{1} \lambda_{2} x\right) .
\end{aligned}
$$

Setting $\lambda_{2}=1$, the term in the parentheses has discriminant in $\lambda_{1}$ equal to

$$
16\left(x^{2}+14 x+1\right)^{3} \text {. }
$$

The roots of this polynomial are distinct from 0,1 . If $x$ is a root of this polynomial, then the pencil has only one further singular fiber which is then necessarily a cusp. Otherwise the pencil has two further singular fibers which are fishtails.

Finally, the generic case is when next to $I_{0}^{*}$ and the $I_{2}$ fiber there are four $I_{1}$ fibers. To get an example with these singular fibers, pick $\sigma_{1}=(u-v)^{2}(u+v)^{2}, \sigma_{2}=u^{2} v(u+2 v)$, giving rise to fibers of type $I_{2}, I_{1}$ respectively. Then the generic curve in the pencil has discriminant

$$
\Delta=\lambda_{1} \lambda_{2}^{2}\left(768 \lambda_{1}^{3}-832 \lambda_{1}^{2} \lambda_{2}+325 \lambda_{1} \lambda_{2}^{2}-32 \lambda_{2}^{3}\right) \text {. }
$$

The cubic polynomial in parentheses admits three distinct roots, each providing a value of $\left[\lambda_{1}: \lambda_{2}\right]$ for which the corresponding curve in the fibration is singular. By the classification, these singular curves are of type $I_{1}$. 
It is easy to see that if the fibration contains (next to $I_{0}^{*}$ ) a fiber which is $I_{4}, I_{3}, I_{2}$ or type $I V$ or $I I I$ (their common property being that they contain rational (-2)-curves), then the pencil in $\mathbb{F}_{2}$ either contains curves with more than one components (i.e., unions of two sections of the ruling), or nodal/cuspidal curves with their singular points on a chosen fiber $F_{i}$. These cases comprise the first three rows of the list summarized in Table 1, and were all considered above.

If no such curves are present in the pencil, then the singular curves in the pencil are either cusps or nodal curves, with their singular points away from the chosen fibers. There are four possibilities, listed in the fourth row of Table 1: next to the $I_{0}^{*}$ fiber we can have (a) three cusps, (b) two cusps and two fishtails, (c) one cusp and four fishtails and finally (d) six fishtails. Clearly, the last case is the generic. In the following we will discuss some cases left open above. We will only discuss pencils in the Hirzebruch surface $\mathbb{F}_{2}$.

Example 6.12 (three or two cusp fibers). It is relatively easy to find a pencil giving rise to a fibration with at least two cusps (next to $\left.I_{0}^{*}\right)$ : consider the double sections coming from the homogeneous degree- 4 polynomials $u^{3} v$ and $(u+x v)^{3}(u+v)$ with parameter $x \neq 0,1$. If we take the fibers over the points $\left[u_{i}: 1\right] \in \mathbb{C} P^{1}$ where $u_{i}$ solves $(u+x)^{3}(u+1)=u^{3}$, we get at least two cusps.

We are in cases (a) or (b) above. The actual case depends on the value of $x$. Indeed the general polynomial of the pencil has discriminant in $u$ (setting $v=1$ ) equal to

$$
\begin{gathered}
-\lambda_{1}^{2} \lambda_{2}^{2} x^{6}\left(27 \lambda_{1}^{2}+27 \lambda_{2}^{2}+54 \lambda_{1} \lambda_{2}+27 \lambda_{2}^{2} x^{4}-108 \lambda_{2}^{2} x^{3}+4 \lambda_{1} \lambda_{2} x^{3}\right. \\
\left.+162 \lambda_{2}^{2} x^{2}-18 \lambda_{1} \lambda_{2} x^{2}-108 \lambda_{2}^{2} x+216 \lambda_{1} \lambda_{2} x\right) .
\end{gathered}
$$

Setting $\lambda_{2}=1$, the term in the parentheses has discriminant in $\lambda_{1}$ equal to

$$
16 x(x-9)^{2}(x+3)^{3} .
$$

The roots of this polynomial are -3 and 9 . If $x$ is equal to one of these values, then the pencil has only one further singular fiber which is then necessarily a cusp. Otherwise the pencil has two further singular fibers which are fishtails.

Example 6.13 (one cusp fiber). In a similar manner, taking $u^{3} v$ and $(u-v)^{2}(u+v)(u+2 v)$ we get a fibration with a cusp and a fishtail fiber next to the $I_{0}^{*}$ fiber; the discriminant of the generic curve in the pencil is given by

$$
\Delta=-\lambda_{1} \lambda_{2}^{2}\left(108 \lambda_{1}^{3}+320 \lambda_{1}^{2} \lambda_{2}+1827 \lambda_{1} \lambda_{2}^{2}+864 \lambda_{2}^{3}\right) .
$$

As the cubic in parentheses admits three distinct roots, the remaining fibers are all $I_{1}$ fibers, giving the example of a cusp together with four fishtails.

Example 6.14 (six fishtails). The generic case in this situation is simply six $I_{1}$ fibers next to $I_{0}^{*}$. To construct an example, set $\sigma_{1}=(u-v)^{2}(u+v)(u+3 v), \sigma_{2}=u^{2} v(u+2 v)$, both giving rise to a type $I_{1}$ fiber. The discriminant of the generic curve in the pencil reads as

$$
\Delta=-\lambda_{1} \lambda_{2}\left(24576 \lambda_{1}^{4}-8272 \lambda_{1}^{3} \lambda_{2}+3768 \lambda_{1}^{2} \lambda_{2}^{2}-517 \lambda_{1} \lambda_{2}^{3}+96 \lambda_{2}^{4}\right) .
$$

It can be shown that the quartic polynomial in parentheses has non-vanishing discriminant, therefore $\Delta$ has four distinct roots $\left[\lambda_{1}: \lambda_{2}\right]$, giving rise to four further fibers of type $I_{1}$.

Proof of Theorem 1.7. The combination of the examples given in this section provide the proof of the theorem. 


\section{Acknowledgments}

The authors acknowledge support of NKFIH through the Élvonal (Frontier) program, with grant KKP126683. The third author was also supported by the grant NKFIH 120697. The authors would like to thank the anonymous referees for many helpful comments, suggestions and corrections.

\section{References}

[1] Alekseev A., Malkin A., Meinrenken E., Lie group valued moment maps, J. Differential Geom. 48 (1998), 445-495, arXiv:dg-ga/9707021.

[2] Biquard O., Boalch P., Wild non-abelian Hodge theory on curves, Compos. Math. 140 (2004), 179-204, arXiv:math.DG/0111098.

[3] Boalch P., Hyperkahler manifolds and nonabelian Hodge theory of (irregular) curves, arXiv:1203.6607.

[4] Boalch P., Simply-laced isomonodromy systems, Publ. Math. Inst. Hautes Études Sci. 116 (2012), 1-68, arXiv:1107.0874.

[5] Boalch P., Geometry and braiding of Stokes data; fission and wild character varieties, Ann. of Math. 179 (2014), 301-365, arXiv:1111.6228.

[6] Boalch P., Yamakawa D., Twisted wild character varieties, arXiv:1512.08091.

[7] Crawley-Boevey W., On matrices in prescribed conjugacy classes with no common invariant subspace and sum zero, Duke Math. J. 118 (2003), 339-352, arXiv:math.RA/0103101.

[8] de Cataldo M.A.A., Hausel T., Migliorini L., Topology of Hitchin systems and Hodge theory of character varieties: the case $A_{1}$, Ann. of Math. 175 (2012), 1329-1407, arXiv:1004.1420.

[9] Friedman R., Morgan J.W., Smooth four-manifolds and complex surfaces, Ergebnisse der Mathematik und ihrer Grenzgebiete (3), Vol. 27, Springer-Verlag, Berlin, 1994.

[10] Gong C., Lu J., Tan S.-L., On families of complex curves over $\mathbb{P}^{1}$ with two singular fibers, Osaka J. Math. 53 (2016), 83-99.

[11] Hitchin N.J., The self-duality equations on a Riemann surface, Proc. London Math. Soc. 55 (1987), $59-126$.

[12] Hitchin N.J., Lie groups and Teichmüller space, Topology 31 (1992), 449-473.

[13] Ivanics P., Stipsicz A., Szabó S., Two-dimensional moduli spaces of rank 2 Higgs bundles over $\mathbb{C} P^{1}$ with one irregular singular point, J. Geom. Phys. 130 (2018), 184-212, arXiv:1604.08503.

[14] Ivanics P., Stipsicz A., Szabó S., Hitchin fibrations on moduli of irregular Higgs bundles and motivic wallcrossing, J. Pure Appl. Algebra 223 (2019), 3989-4064, arXiv:1710.09922.

[15] Kodaira K., On compact analytic surfaces. II, Ann. of Math. 77 (1963), 563-626.

[16] Kraft H., Procesi C., Closures of conjugacy classes of matrices are normal, Invent. Math. 53 (1979), $227-247$.

[17] Kronheimer P.B., Nakajima H., Yang-Mills instantons on ALE gravitational instantons, Math. Ann. 288 (1990), 263-307.

[18] Miranda R., Persson's list of singular fibers for a rational elliptic surface, Math. Z. 205 (1990), $191-211$.

[19] Nitsure N., Moduli space of semistable pairs on a curve, Proc. London Math. Soc. 62 (1991), 275-300.

[20] Persson U., Configurations of Kodaira fibers on rational elliptic surfaces, Math. Z. 205 (1990), 1-47.

[21] Simpson C., Harmonic bundles on noncompact curves, J. Amer. Math. Soc. 3 (1990), 713-770.

[22] Simpson C., The dual boundary complex of the $\mathrm{SL}_{2}$ character variety of a punctured sphere, Ann. Fac. Sci. Toulouse Math. 25 (2016), 317-361, arXiv:1504.05395.

[23] Stipsicz A.I., Szabó Z., Szilárd A., Singular fibers in elliptic fibrations on the rational elliptic surface, Period. Math. Hungar. 54 (2007), 137-162.

[24] Szabó S., The birational geometry of unramified irregular Higgs bundles on curves, Internat. J. Math. 28 (2017), 1750045, 32 pages, arXiv:1502.02003.

[25] Szabó S., Perversity equals weight for Painlevé spaces, arXiv:1802.03798.

[26] Tan S.-L., Chern numbers of a singular fiber, modular invariants and isotrivial families of curves, Acta Math. Vietnam. 35 (2010), 159-172. 